\title{
High-Performance Control of Piezoelectric Tube Scanners
}

\author{
B. Bhikkaji, M. Ratnam, Andrew J. Fleming, and S. O. Reza Moheimani, Senior Member, IEEE
}

\begin{abstract}
In this paper, a piezoelectric tube of the type typically used in scanning tunneling microscopes (STMs) and atomic force microscopes (AFMs) is considered. Actuation of this piezoelectric tube is hampered by the presence of a lightly damped low-frequency resonant mode. The resonant mode is identified and damped using a positive velocity and position feedback (PVPF) controller, a control technique proposed in this paper. Input signals are then shaped such that the closed-loop system tracks a raster pattern. Normally, piezoelectric tubes are actuated using voltage amplifiers. Nonlinearity in the form of hysteresis is observed when actuating the piezoelectric tubes at high amplitudes using voltage amplifiers. It has been known for some time that hysteresis in piezoelectric actuators can be largely compensated by actuating them using charge amplifiers. In this paper, high-amplitude actuation of a piezoelectric tube is achieved using a charge amplifier.
\end{abstract}

Index Terms-Charge amplifiers, damping, feedback control, hysteresis, piezoelectric tube, raster pattern, resonant mode, system identification.

\section{INTRODUCTION}

$\mathbf{S}$ CANNING tunneling microscopes (STMs) and atomic force microscopes (AFMs) are used extensively in diverse areas of science such as crystallography, cell biology, etc., [1]. When used at extreme magnifications, they are capable of generating topographical maps of solid surfaces at micro to atomic resolution. In both STMs and AFMs, a probe is placed in close proximity, typically a few nanometers, to the material surface for which a topographical map is desired. The given material surface is scanned by either moving the probe or the sample in a raster pattern, so that the probe interacts with the entire region of interest [1]. In many commercially available STMs and AFMs, scanning is performed using a piezoelectric tube. Depending on the make, either the probe is attached to the piezoelectric tube or the sample is placed on piezoelectric tube and the piezoelectric tube is actuated in a raster pattern.

One of the advantages of using piezoelectric tubes is that under certain experimental conditions their dynamics can be well approximated by linear models (see [2]-[8]). The linear models normally reveal the presence of lightly damped resonance modes, which make the piezoelectric tubes susceptible

Manuscript received September 20, 2006; revised November 20, 2006. Manuscript received in final form May 1, 2007. Recommended by Guest Editor S. Davasia. This work was supported by the ARC Center of Excellence for Complex Dynamic Systems and Control.

The authors are with the School of Electrical Engineering and Computer Science, University of Newcastle, Callaghan, NSW 2308, Australia (e-mail: bharath.bhikkaji@newcastle.edu.au; mratnam@ee.newcastle.edu.au; andrew.j. fleming@ee.newcastle.edu.au; reza.moheimani@newcastle.edu.au).

Color versions of one or more of the figures in this paper are available online at http://ieeexplore.ieee.org.

Digital Object Identifier 10.1109/TCST.2007.902947 to mechanical vibrations. Nonlinear phenomenon such as creep and hysteresis also become visible when actuating the tube using low-frequency and high-amplitude inputs, respectively, [9], [10]. In such scenarios, the linear approximations become inadequate. The objective of this paper is to perform fast scans using a piezoelectric tube. The main impediments to fast scanning are the presence of mechanical vibrations and hysteresis.

The use of feedback control to compensate for the vibrations in the piezoelectric tubes has been investigated by several authors [2], [4], [6]. Controllers such as the proportional-integral (PI), proportional-integral-integral (PII), and the proportional-integral-differential (PID), though easily implementable, when used alone, suffer from low tracking bandwidth [6]. Moreover, due to their structure, the chances of them exciting the unmodeled higher order modes of the tube are also high. In order to have good robustness properties, robust control approaches like $\mathcal{H}_{\infty}$ control have also been used [6]. It is desirable to have controllers that are easy to implement, robust, have a roll-off in the high frequency regions, and also damp the system.

Caughey and coauthors introduced a control technique known as the positive position feedback (PPF) control [11], [12] to suppress the mechanical vibrations in flexible structures. The parametric structure of the PPF controller is such that its frequency response rolls-off of as $1 / \omega^{2}$ at high frequencies. Several authors have successfully implemented PPF controllers [13]-[18]. In [17], Ratnam et al. designed a PPF controller to damp vibrations in a piezoelectric tube scanner. Therein, it was noted that using a PPF controller poles of the closed-loop system cannot be placed at any given set of points in the left half plane. In order to allow this flexibility, PPF controller is modified into a positive velocity and position feedback (PVPF) controller. Thus, PVPF is a control technique introduced in this paper to damp the first resonance of a piezoelectric tube.

Nonlinearity in the form of hysteresis becomes visible when piezoelectric tubes are actuated using voltage signals of high amplitudes. At low range scans (i.e., when actuating a piezoelectric tube with voltage signals of low amplitude), hysteresis can be neglected. A popular approach to compensate for hysteresis is to model it as a nonlinear function $H(\cdot)$ and then eliminate it by cascading its inverse $H^{-1}(\cdot)$ with piezoelectric tube actuator (see [19]-[22]). Feedback control schemes have also been used to compensate for hysteresis in [4], [6], and [23]. Since the late 1980s, it has been known that actuating piezoelectric transducers with current or charge sources rather than voltage sources significantly reduces hysteresis [24]. In fact, it has been noted that using current or charge sources, at least a five-fold reduction in hysteresis can be achieved [25].

Nonlinear modeling of hysteresis has always been a tedious task. Even in cases where the hysteresis is rate independent constructing a good model is both complex and computationally 
involved [26]. In cases where the hysteresis is rate dependent, as in the case of piezoelectric materials, current modeling schemes offer little help. The use of charge or current sources does not necessitate the modeling of hysteresis. However, herein, the problem lies with the instrumentation of the charge or current sources, as they are not commercially available. Invariably, the only resort is to make a "homemade" charge or current source. In [27] and [28], the authors have designed a charge source for the general purpose of exciting piezoelectric actuators without encountering hysteresis. In this paper, this charge amplifier is used for applying signals to the piezoelectric tube in order to avoid hysteresis.

In this paper, a piezoelectric tube of the type typically used in the scanning unit of STMs and AFMs is considered. In Section II, linear models are constructed for the tube and the resonant mode of interest is identified. The need for designing a feedback controller is motivated in Section III. The concept of PVPF control is introduced in Section IV. In Section V, a PVPF controller is designed and the closed-loop system is actuated in a raster pattern. In Section VI, the existence of hysteresis is discussed. The circuit topology of the charge amplifier and its effect on the dynamics of the PZT scanner are presented therein. Identification, modeling, and PVPF control of the PZT scanner, when using the charge amplifier, are also presented in Section VI.

\section{SYSTEM IDENTIFICATION}

In this section, experiments are performed on the piezoelectric tube by exciting it with specific input signals and recording the corresponding outputs. A model is then fitted for each recorded input/output (I/O) data.

\section{A. Experimental Setup}

A piezoelectric tube scanner is a thin-walled cylindrical tube made of piezoelectric material. Its inner and outer walls are finely coated with a layer of silver. The silver coating acts as the electrodes of the scanner. The outer electrode of the piezoelectric tube scanner is axially quartered into four equal sections. Conventionally, a pair of opposite quartered electrodes are referred to as the $x-x$ electrodes and the other pair is referred to as the $y-y$ electrodes. Good illustrations of the tube can be found in [1], [17], and [28].

A jig is constructed to hold the piezoelectric tube along the $z$-axis. A small aluminum cube is bonded to the upper end of the tube. This cube represents the seat where the materials that need to be scanned are placed. The heads of two ADE Technologies 4810 capacitive sensors are placed in close proximity to the adjacent faces of the aluminum cube in the $x$ and $y$ directions, respectively (see Fig. 1). The inner electrode of the piezoelectric tube is grounded. One electrode each of the $x-x$ and $y-y$ pairs, referred as $x^{+}$and $y^{+}$, respectively, are chosen as the input ends of the piezoelectric tube, and the corresponding opposite ends, referred to as $x^{-}$and $y^{-}$, respectively, are chosen as the output ends. The whole setup consisting of the piezoelectric tube with the bonded aluminum cube and the heads of the capacitive sensors, is placed in a specially constructed cylindrical enclosure (see Fig. 2). The cylindrical enclosure protects the experimental setup from external noise.

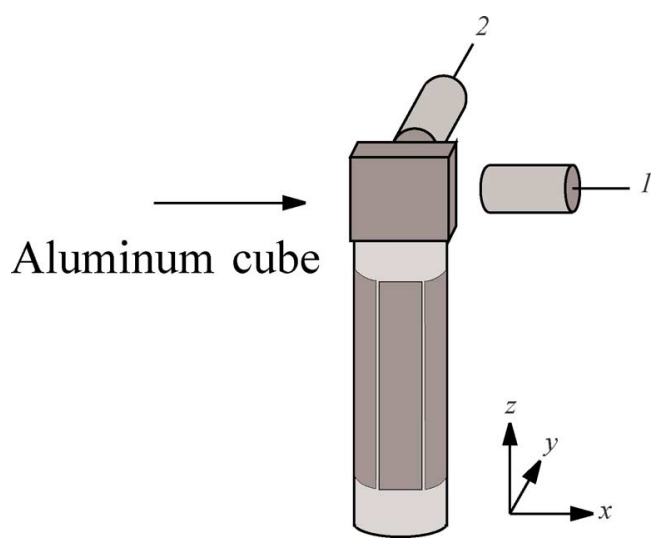

Fig. 1. Schematic diagram of the experimental setup: 1 and 2 are the heads of the capacitive sensors.

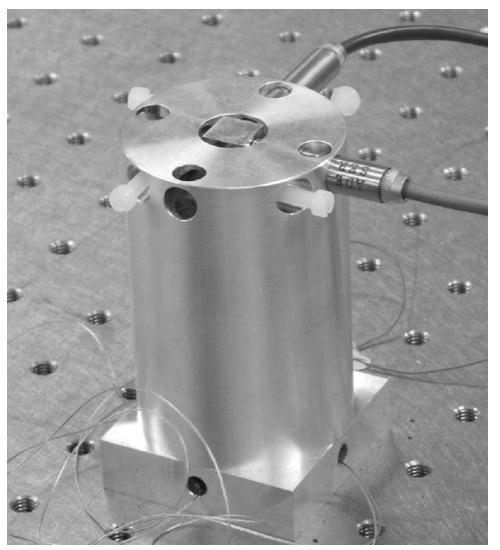

Fig. 2. Piezoelectric tube mounted inside an aluminum shield. The $x$-axis capacitive sensor is shown secured at right angles to a cube mounted onto the tube tip. And the $y$-axis capacitive sensor is secured at right angles to the perpendicular face of the aluminum cube.

When voltage signals $V_{x^{+}}$and $V_{y^{+}}$are applied at the electrodes $x^{+}$and $y^{+}$, respectively, the piezoelectric tube deforms inducing voltages $V_{x^{-}}$and $V_{y^{-}}$at the output electrodes $x^{-}$ and $y^{-}$, respectively. The induced voltages $\left[V_{x^{-}}, V_{y^{-}}\right]^{\top}$ are recorded and taken as outputs. Furthermore, due to the deformation of the tube, the capacitance between the aluminum cube and the heads of the capacitive sensors change. The change in the capacitance is used by the capacitive sensors to measure the distance between their respective heads and the aluminum cube. This distance, denoted by $C=\left[d_{x}, d_{y}\right]^{\top}$, is also recorded as an output. The outputs $d_{x}$ and $d_{y}$ act as indicators of the lateral displacements of the tube in the $x$ and $y$ directions, respectively. In summary, the piezoelectric tube setup is interpreted as a linear system incorporating two linear subsystems $V$ ( $V$ denoting voltage) and $C$ ( $C$ denoting the capacitance). With both $V$ and $C$ having the same inputs $\left[V_{x^{+}}, V_{y^{+}}\right]^{\top}$, but different outputs $\left[V_{x^{-}}, V_{y^{-}}\right]^{\top}$ and $\left[d_{x}, d_{y}\right]^{\top}$, respectively (see Fig. 3).

Quantitatively stated, the piezoelectric tube setup is modeled as having linear subsystems $V$ and $C$. Subsystem $V$ is of the form

$$
Y_{v}(s) \triangleq G_{v v}(s) U(s)
$$




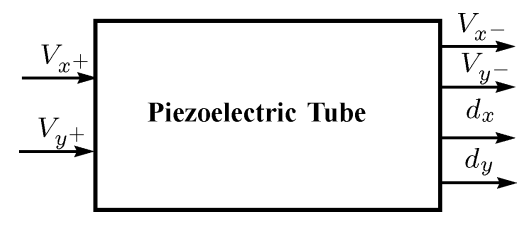

Fig. 3. I/O diagram.

with $Y_{v}(s)$ denoting the Laplace transform of the voltages $\left[V_{x^{-}}, V_{y^{-}}\right], U(s)$ denoting the Laplace transform of the input voltages $\left[V_{x^{+}}, V_{y^{+}}\right]^{\top}$, and

$$
G_{v v}(s)=\left[\begin{array}{ll}
G_{x x}^{(v)}(s) & G_{x y}^{(v)}(s) \\
G_{y x}^{(v)}(s) & G_{y y}^{(v)}(s)
\end{array}\right]
$$

being a $2 \times 2$ matrix of transfer-functions. The subscript $v v$ in $G_{v v}(s)$ denotes the fact that both the inputs and outputs are voltages. The subsystem $C$ is of the form

$$
Y_{d}(s) \triangleq G_{d v}(s) U(s)
$$

where $Y_{d}(s)$ is the Laplace transform of the capacitive sensor output signals $\left[d_{x}, d_{y}\right]^{\top}$

$$
G_{d v}(s)=\left[\begin{array}{ll}
G_{d_{x} x}^{(v)}(s) & G_{d_{y} x}^{(v)}(s) \\
G_{d_{x} y}^{(v)}(s) & G_{d_{y} y}^{(v)}(s)
\end{array}\right]
$$

is a $2 \times 2$ matrix of transfer functions. In this case, the subscript $d v$ in $G_{d v}(s)$ denotes the fact that the inputs are voltages and outputs are displacements.

Since the tube, along with the bonded aluminum cube, is symmetric with respect to any plane containing the vertical axis of the tube, in principle, it is expected that $G_{x x}^{(v)}(s)=G_{y y}^{(v)}(s)$ and $G_{x y}^{(v)}(s)=G_{y x}^{(v)}(s)$. Similarly, due to symmetry in the alignment of the capacitive sensors with the faces of the aluminum cube in the $x$ and $y$ directions, it is also expected that $G_{d_{x} x}^{(v)}(s)=G_{d_{y} y}^{(v)}(s)$ and $G_{d_{y} x}^{(v)}(s)=G_{d_{x} y}^{(v)}(s)$.

\section{B. Modeling}

An experiment is performed on the piezoelectric tube by applying swept sine waves to the $x^{+}$and $y^{+}$electrodes, and recording the corresponding voltage outputs at $x^{-}$and $y^{-}$and the capacitive sensor outputs $d_{x}$ and $d_{y}$ using an HP 35670A dual channel spectrum analyzer. The spectrum analyzer is also used to process the recorded $\mathrm{I} / \mathrm{O}$ data and to obtain frequency response functions (FRF) $G_{v v}(i \omega)$ and $G_{d v}(i \omega)$, respectively.

In Fig. 4, the magnitude of the FRFs $G_{x x}^{(v)}(i \omega), G_{y y}^{(v)}(i \omega)$, $G_{x y}^{(v)}(i \omega)$, and $G_{y x}^{(v)}(i \omega)$ are plotted. It can be noted that $\left|G_{x x}^{(v)}(i \omega)\right| \approx\left|G_{y y}^{(v)}(i \omega)\right|$. However, the same cannot be said for the cross coupling terms $G_{x y}^{(v)}(i \omega)$ and $G_{y x}^{(v)}(i \omega)$. The magnitude plots of the cross terms in Fig. 4, suggest small but noticeable differences in frequency regions near the resonance frequency of the tube.

Similarly, in Fig. 5 the magnitude of the FRFs $G_{d_{x} x}^{(v)}(i \omega)$, $G_{d_{y} x}^{(v)}(i \omega), G_{d_{x} y}^{(v)}(i \omega)$, and $G_{d_{y} y}^{(v)}(i \omega)$ are plotted. Here too $\left|G_{d_{x} x}^{(v)}(i \omega)\right| \approx\left|G_{d_{y} y}^{(v)}(i \omega)\right|$, and the cross coupling terms
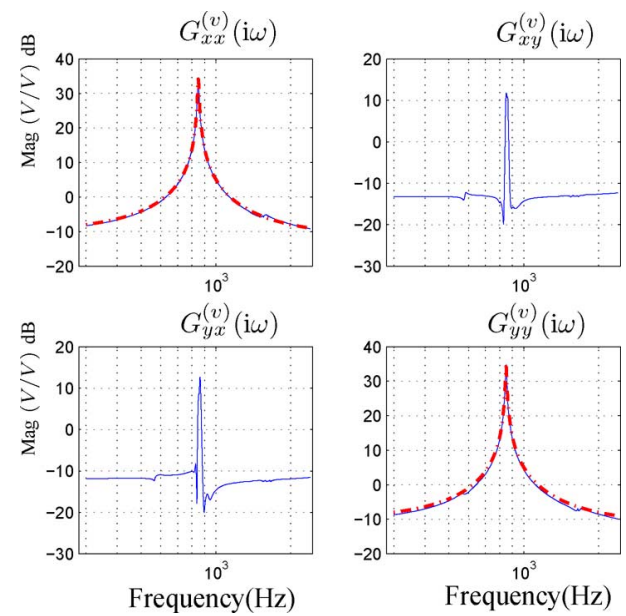

Fig. 4. Magnitude plots of the data (solid line) along with the corresponding parametric models (dashed dotted line).
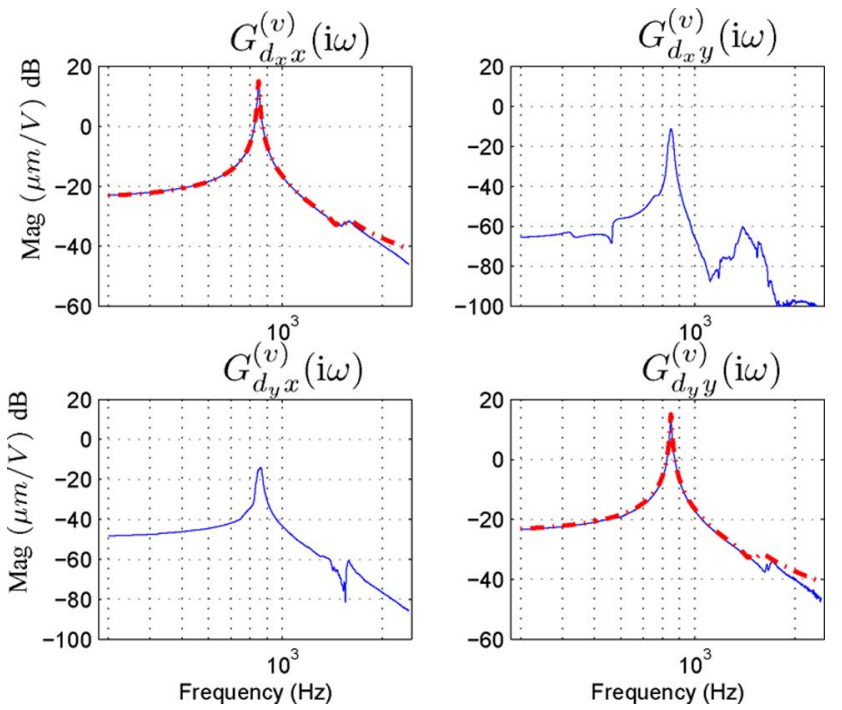

Fig. 5. Magnitude plots of the data (solid line) along with the corresponding parametric models (dashed dotted line).

$G_{d_{y} x}^{(v)}(i \omega)$ and $G_{d_{x} y}^{(v)}(i \omega)$ differ from each other. Note that the cross coupling terms $G_{d_{y} x}^{(v)}(i \omega)$ and $G_{d_{x} y}^{(v)}(i \omega)$ are negligible in magnitude, except at frequencies around the resonance. In other words, input $V_{x^{+}}$will have little effect on the capacitive sensor output $d_{y}$ in the $y$ direction, unless the frequencies close to the resonance are excited. A similar argument applies to the cross coupling in the other dimension.

Apart from the oscillations in the $x$ and $y$ directions, the tube also oscillates along its circumference and the $z$-axis. These oscillatory modes are referred to as the torsional mode and the piston mode, respectively. Both these modes contribute significantly to the cross coupling terms in the voltage subsystem $V$. However, these modes have little effect on the capacitive sensor measurements.

In summary, the direct terms $G_{x x}^{(v)}(i \omega), G_{y y}^{(v)}(i \omega), G_{d_{x} x}^{(v)}(i \omega)$, and $G_{d_{y} y}^{(v)}(i \omega)$ are in agreement with the expectations arising due to symmetry. However, the cross coupling terms do not concur with those expectations. The discrepancies observed in the cross 
TABLE I

PARAMETER VALUES OF THE TRANSFER-FUNCTIONS $G_{x x}^{(v)}(s), G_{y y}^{(v)}(s), G_{d_{x} x}^{(v)}(s), \operatorname{AND} G_{d_{y} y}^{(v)}(s)$

\begin{tabular}{|c|c|}
\hline$k_{1}$ & $1.6711 \times 10^{7}$ \\
\hline $2 \sigma \omega$ & 60.2 \\
\hline$\omega^{2}$ & $2.8488 \times 10^{7}$ \\
\hline$d_{1}$ & $-2.723 \times 10^{-1}$ \\
\hline$c_{1}$ & 0.0055 \\
\hline$c_{2}$ & -112.3 \\
\hline$c_{3}$ & $1.807 \times 10^{6}$ \\
\hline
\end{tabular}

coupling terms could be due to manufacturing defects, e.g., tube eccentricity or engineering tolerances such as extra dynamics added by the wires attached to the electrodes of the tube or extra dynamics added by the glue that was used to bond the tube to its base and to bond the aluminum cube on its top or due to misalignment or noncentric mounting of the aluminum cube.

Since the FRFs $G_{x x}^{(v)}(i \omega), G_{y y}^{(v)}(i \omega), G_{d_{x} x}^{(v)}(i \omega)$, and $G_{d_{y} y}^{(v)}(i \omega)$ each have a resonant peak, in the frequency range of concern, second-order models are fit to them using standard techniques. The following models were found to fit the data:

$$
\begin{aligned}
G_{x x}^{(v)}(s) & =G_{y y}^{(v)}(s)=\frac{k_{1}}{s^{2}+2 \sigma \omega s+\omega^{2}}+d_{1} \\
G_{d_{x} x}^{(v)}(s) & =G_{d_{y} y}^{(v)}(s)=\frac{c_{1} s^{2}+c_{2} s+c_{3}}{s^{2}+2 \sigma \omega s+\omega^{2}}
\end{aligned}
$$

where the model parameters are as tabulated in Table I.

In Figs. 4 and 5, the magnitude of the parametric fits (5) and (6) are plotted along with their respective data. Parametric fits for the cross coupling terms $G_{x y}^{(v)}(s), G_{y x}^{(v)}(s), G_{d_{y} x}^{(v)}(s)$, and $G_{d_{x} y}^{(v)}(s)$ are not presented here as they are not used for actuating the tube. It can be inferred from Figs. 4 and 5 that the parametric models fit the data reasonably well in the frequency regions plotted. Details on how the models are estimated are not presented here as the models are of low order and their estimation is straightforward.

\section{FEED-ForwARD CONTROL}

As mentioned earlier, our goal is to actuate the piezoelectric tube in a raster pattern. Therefore, a desired trajectory for the piezoelectric tube would be to repeatedly trace straight lines back and forth in the $x$ direction, while slowly increasing its position in the $y$ direction. A common practice to track such a trajectory is to apply a triangular signal to the $x^{+}$electrode and a "very slowly" increasing ramp signal to the $y^{+}$. In fact, to have a good scan of the surface the changes in the $y$ direction must be "quasi-static" with respect to the changes in the $x$ direction. Normally for illustration purposes, the slowly varying ramp in the $y^{+}$electrode is either replaced by a dc signal or assumed to be earthed or open circuited (see [2]-[4]).

In Figs. 6 and 7, the capacitive sensor responses $d_{x}$ and $d_{y}$, respectively, to a triangle wave input of $30 \mathrm{~V}$ and $40 \mathrm{~Hz}$ at the $x^{+}$ electrode with the $y^{+}$electrode being earthed is plotted. Note that the capacitive sensor output $d_{x}$ is not exactly a triangular waveform, but appears to be equal to a triangular waveform plus certain periodic corrugations. This implies that the lateral displacement of the tube along the $x$-axis is not really a straight

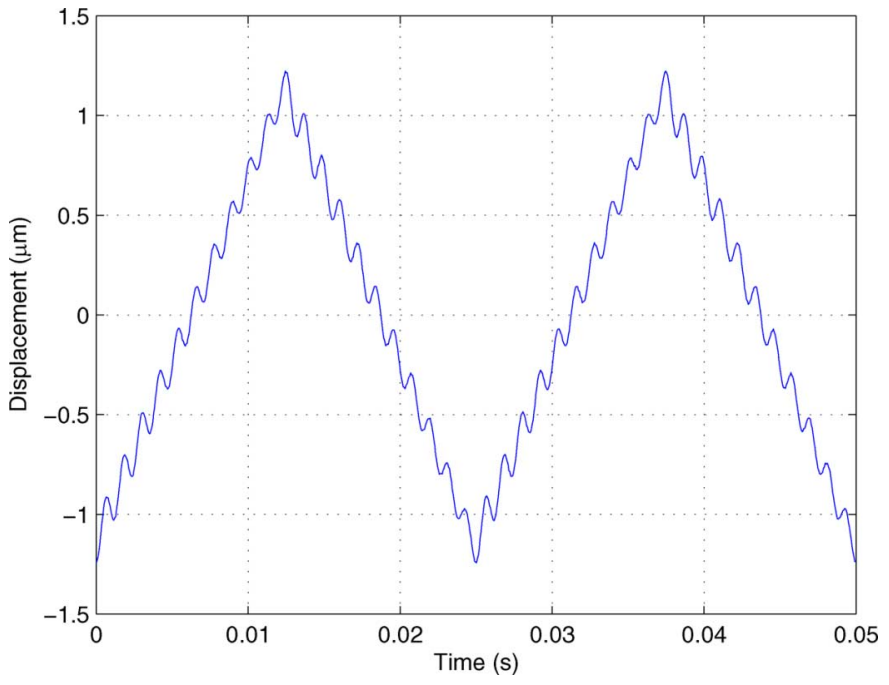

Fig. 6. Response recorded by the capacitive sensor $d_{x}$ for a triangular waveform input with an amplitude of $30 \mathrm{~V}$ and a fundamental frequency of $40 \mathrm{~Hz}$.

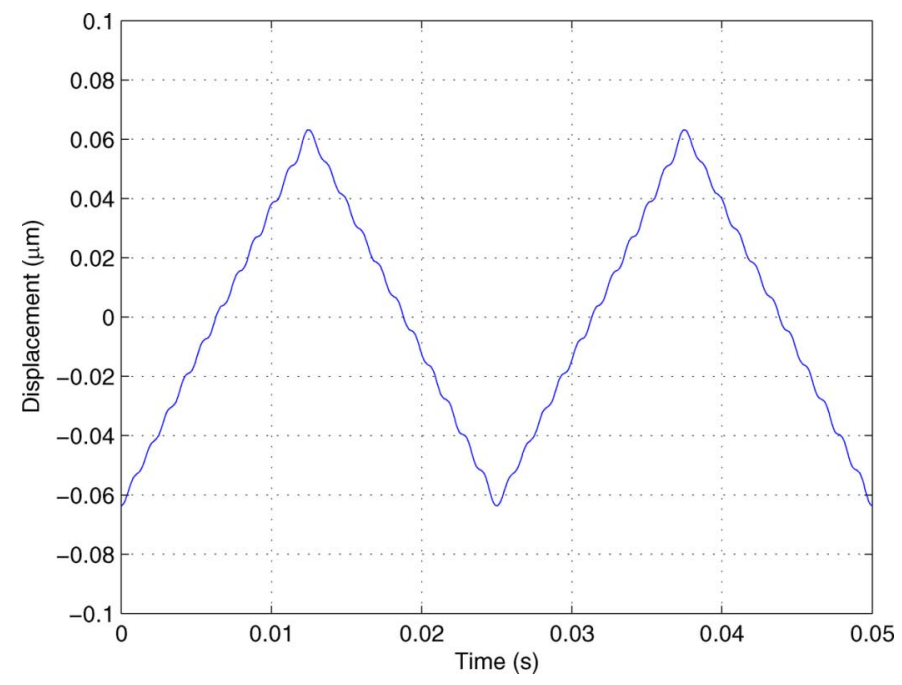

Fig. 7. Response recorded by the capacitive sensor $d_{y}$ for a triangular waveform input with an amplitude of $30 \mathrm{~V}$ and a fundamental frequency of $40 \mathrm{~Hz}$.

line but rather a highly corrugated straight line. The distortion in the capacitive sensor output $d_{x}$ is due to the amplification of the 21 st and the 23rd harmonics of the triangular waveform which are close to the resonance frequency of the piezoelectric tube. It is apparent from Fig. 7 that $d_{y}$ is also a periodic triangular waveform but with an amplitude that is negligible when compared with the amplitude of $d_{x}$. This is not surprising due to the weak cross coupling that exists between the input $V_{x^{+}}$and the output $d_{y}$.

In general, to eliminate the periodic corrugations in the output at $d_{x}$, two different approaches are taken. In the first approach, instead of using a triangular waveform as input at $x^{+}$, a custom designed input

$$
r(t) \triangleq \sum_{k=1}^{\infty} \frac{a_{k}}{\left|G_{d_{x} x}^{(v)}\left(i \omega_{k}\right)\right|} \sin \left(\omega_{k} t-\phi_{k}\right)
$$




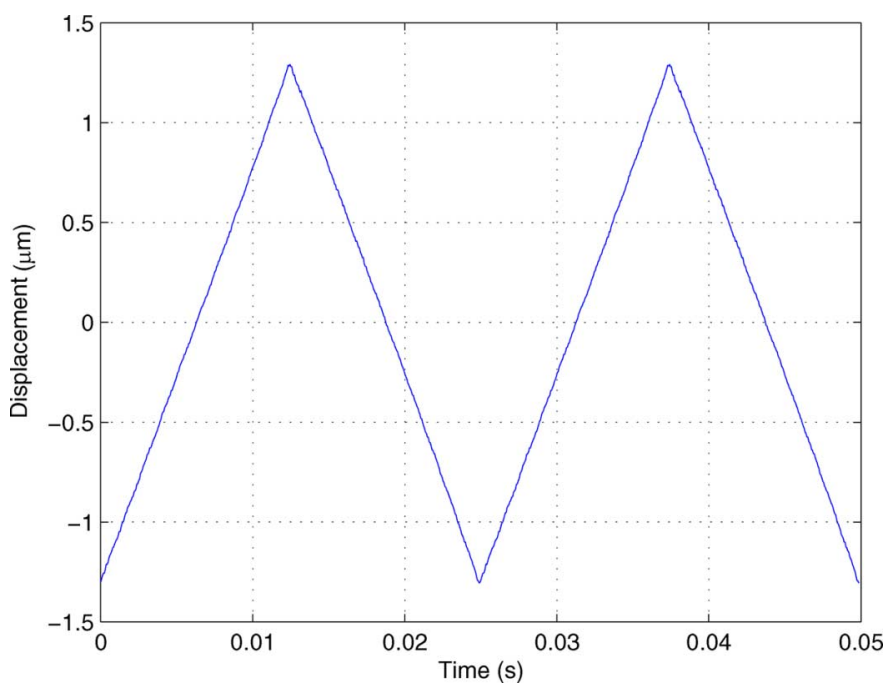

Fig. 8. Response recorded by the capacitive sensor $d_{x}$ for an input $r(t)$ of the form (7) with $f_{d}(t)$ being a triangular waveform with an amplitude of $30 \mathrm{~V}$ and a fundamental frequency of $40 \mathrm{~Hz}$.

where $a_{k}$ and $\omega_{k}$ are such that

$$
f_{d}(t) \triangleq \sum_{k=1}^{\infty} a_{k} \sin \left(\omega_{k} t\right)
$$

is the desired triangular waveform output at $d_{x}$ in the Fourier series form and

$$
\phi_{k} \triangleq \arg G_{d_{x} x}^{(v)}\left(i \omega_{k}\right)
$$

is applied. It is easy to see that applying $r(t)$, (7), at $x^{+}$would give $f_{d}(t)$ at the output $d_{x}$. The second approach is to use a feedback controller that would damp the resonance in $G_{d_{x} x}^{(v)}(s)$, and then input a triangular waveform to the closed-loop system. Note that damping the resonance peaks in $G_{d_{x} x}^{(v)}(s)$ would automatically suppress the amplification of the triangular waveform harmonics that are close to the resonance.

A first look at the two approaches would suggest that the first method is more prudent and simpler than the second. In Fig. 8, the capacitive sensor response $d_{x}$ to an input of the form (7) with $f_{d}(t)$ being the Fourier decomposition of a $30-\mathrm{V}$ amplitude and $40-\mathrm{Hz}$ triangular waveform and $G_{d_{x} x}^{(v)}(s)$ as in (6) is plotted. The capacitive sensor response $d_{x}$ appears to be a smooth triangular waveform. However, this approach is heavily dependent on the correctness of the model $G_{d_{x} x}^{(v)}(s)$, and consequently, suffers from lack of robustness towards model uncertainties. In general, due to wear and tear and other external influences, piezoelectric tube characteristics such as gain and resonance frequency are prone to minor changes or perturbations. In particular, when the system's resonance frequency is perturbed, applying $r(t)$ would not result in a triangular waveform at the output. To illustrate this, in Fig. 9, the response $d_{x}$ of the capacitive senor to the input $r(t)$ is plotted with the resonance of the tube perturbed artificially by placing a nonconducting mass on the bonded aluminum cube. Note that periodic corrugations, similar to the one seen in Fig. 6, appear in Fig. 9 also. Therefore, to minimize the

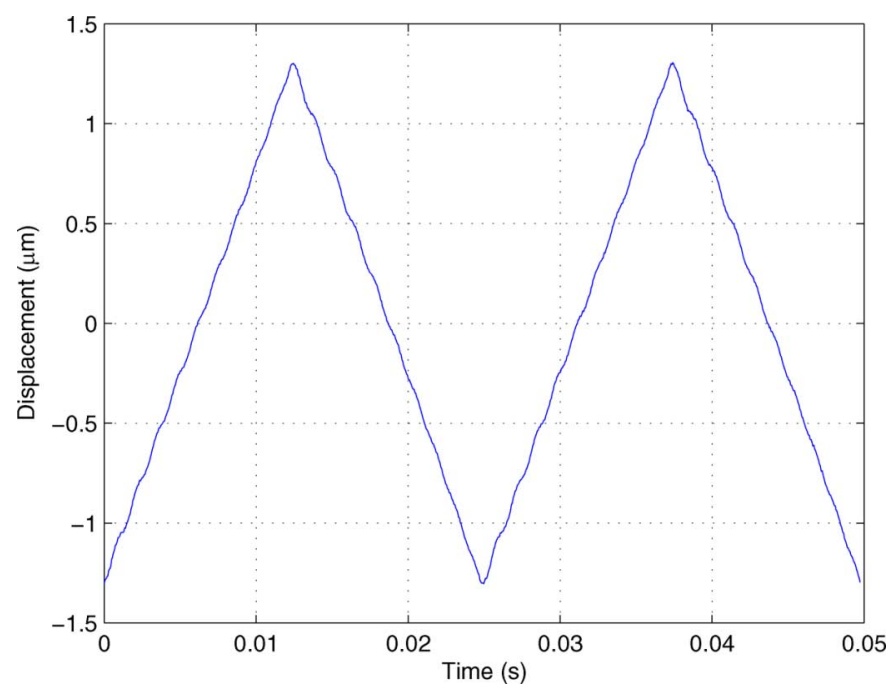

Fig. 9. Response recorded by the capacitive sensor $d_{x}$, for an input $r(t)$ of the form (7) with $f_{d}(t)$ being a triangular waveform with an amplitude of $30 \mathrm{~V}$ and a fundamental frequency of $40 \mathrm{~Hz}$, when the system resonance frequency is perturbed.

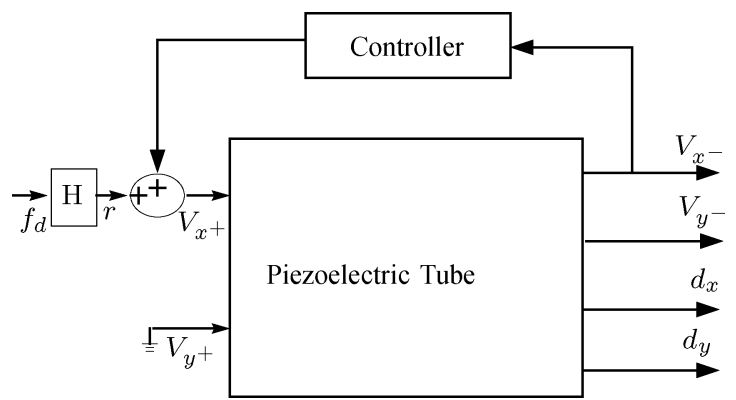

Fig. 10. Closed-loop system along the feed-forward input. Here, $f_{d}$ is the triangular waveform, (8), $r$ denotes the designed input of the form (7) and $\mathrm{H}$ denotes the inverse of the FRF of the closed-loop capacitive sensor response along the $x$-axis.

effects of these perturbations, it is imperative to damp the resonant peak. In this paper, both approaches are adopted. First, a feedback controller is constructed, linking the voltage output $V_{x^{-}}$to the input $V_{x^{+}}$, to damp the vibrations, then an input of the type $r(t),(7)$, is designed for a closed-loop system to get the desired triangular waveform as the output at $d_{x}$ (see Fig. 10).

\section{PVPF CONTROLLER}

In this section, the concept of PVPF control is introduced and a controller of this type is designed to damp the resonant peak in the transfer function $G_{x x}^{(v)}(s)$.

For technical ease, we rewrite $G_{x x}^{(v)}(s)$ in standard secondorder form

$$
\begin{aligned}
\ddot{x}+2 \sigma \omega \dot{x}+\omega^{2} x & =\Psi_{1} u \\
y & =\Psi_{2} x+d u
\end{aligned}
$$

where $\Psi_{1}=1.6711 \times 10^{7}, \Psi_{2}=1$, and $d=-2.723 \times 10^{-1}$ [see (5)].

PVPF controller is defined by

$$
\ddot{z}+2 \xi w \dot{z}+w^{2} z=\Gamma_{1} \dot{v}+\Gamma_{2} v
$$


where $v$ is the input to the controller and $\xi, w, \Gamma_{1}$, and $\Gamma_{2}$ are the design parameters.

If $r(t)$ is the desired system output signal, generally referred to as the reference signal, the corresponding input to the system (10), is set to

$$
u \triangleq z+r
$$

and the controller input is set to

$$
v(t) \triangleq y \text {. }
$$

Using (12) and (13) in (10) and (11), respectively, gives

$$
\begin{aligned}
\ddot{x}+2 \sigma \omega \dot{x}+\omega^{2} x & =\Psi_{1}(z+r) \\
y(t) & =\Psi_{2} x+d(z+r) \\
\ddot{z}+2 \xi w \dot{z}+w^{2} z & =\Gamma_{1} \dot{y}+\Gamma_{2} y
\end{aligned}
$$

respectively.

Note that (15) boils down to

$$
\begin{aligned}
\ddot{z}+2 \xi w \dot{z}+w^{2} z & =\Gamma_{1} \dot{y}+\Gamma_{2} y \\
& =\Gamma_{1}\left(\Psi_{2} \dot{x}+d \dot{z}+d \dot{r}\right)+\Gamma_{2}\left(\Psi_{2} x+d z+d r\right)
\end{aligned}
$$

which implies

$$
\begin{aligned}
\ddot{z}+\left(2 \xi w-\Gamma_{1} d\right) \dot{z}+\left(w^{2}-\Gamma_{2} d\right) z= & \Gamma_{1}\left(\Psi_{2} \dot{x}+d \dot{r}\right) \\
& +\Gamma_{2}\left(\Psi_{2} x+d r\right) .
\end{aligned}
$$

Setting

$$
2 \xi_{1} w_{1} \triangleq 2 \xi w-\Gamma_{1} d
$$

and

$$
w_{1}^{2} \triangleq w^{2}-\Gamma_{2} d
$$

Equation (17) can be rewritten as

$$
\ddot{z}+2 \xi_{1} w_{1} \dot{z}+w_{1}^{2} z=\Gamma_{1}\left(\Psi_{2} \dot{x}+d \dot{r}\right)+\Gamma_{2}\left(\Psi_{2} x+d r\right) .
$$

Clubbing (14) and (20) and rewriting it in matrix form gives (21) shown at the bottom of the page, which can be rewritten as

$$
\begin{array}{r}
{\left[\begin{array}{c}
\ddot{x} \\
\ddot{z}
\end{array}\right]+\left[\begin{array}{cc}
2 \sigma \omega & 0 \\
-\Gamma_{1} \Psi_{2} & 2 \xi_{1} w_{1}
\end{array}\right]\left[\begin{array}{c}
\dot{x} \\
\dot{z}
\end{array}\right]+\left[\begin{array}{cc}
\omega^{2} & -\Psi_{1} \\
-\Gamma_{2} \Psi_{2} & w_{1}^{2}
\end{array}\right]\left[\begin{array}{c}
x \\
z
\end{array}\right]} \\
=\left[\begin{array}{c}
0 \\
\Gamma_{2} d
\end{array}\right] \dot{r}(t)+\left[\begin{array}{c}
\Psi_{1} \\
\Gamma_{1} d
\end{array}\right] r(t) .
\end{array}
$$

Note that the system output is equal to

$$
y(t)=\left[\begin{array}{ll}
\Psi_{2} & d
\end{array}\right]\left[\begin{array}{l}
x \\
z
\end{array}\right]+d r(t)
$$

Laplace transform of (22) and (23) gives

$$
\begin{aligned}
& {\left[\begin{array}{cc}
s^{2}+2 \sigma \omega s+\omega^{2} & -\Psi_{1} \\
-\left(\Gamma_{1} \Psi_{2} s+\Gamma_{2} \Psi_{2}\right) & s^{2}+2 \xi_{1} w_{1}+w_{1}^{2}
\end{array}\right]\left[\begin{array}{l}
x(s) \\
z(s)
\end{array}\right]} \\
& =\left[\begin{array}{c}
\Psi_{1} \\
\Gamma_{2} d s+\Gamma_{1} d
\end{array}\right] r(s) y(s)=\left[\Psi_{2} d\right]\left[\begin{array}{l}
x(s) \\
z(s)
\end{array}\right]+d r(s) .
\end{aligned}
$$

It is evident from (24) that poles of the closed-loop system are the roots of the determinant of the polynomial matrix

$$
A \triangleq\left[\begin{array}{cc}
s^{2}+2 \sigma \omega s+\omega^{2} & -\Psi_{1} \\
-\left(\Gamma_{1} \Psi_{2} s+\Gamma_{2} \Psi_{2}\right) & s^{2}+2 \xi_{1} w_{1}+w_{1}^{2}
\end{array}\right]
$$

Furthermore, it is easy to check that

$$
\begin{aligned}
P(s) \triangleq & \operatorname{det}(A) \\
= & \left(s^{2}+2 \sigma \omega s+\omega^{2}\right)\left(s^{2}+2 \xi_{1} w_{1}+w_{1}^{2}\right)-\Psi_{1} \Psi_{2}\left(\Gamma_{1} s+\Gamma_{2}\right) \\
= & s^{4}+\left(2 \sigma \omega+2 \xi_{1} w_{1}\right) s^{3}+\left(\omega^{2}+2 \sigma \omega 2 \xi_{1} w_{1}+w_{1}\right) s^{2} \\
& +\left(2 \sigma \omega w_{1}^{2}+2 \xi_{1} w_{1} \omega^{2}-\Psi \Gamma_{1}\right) s+\omega^{2} w_{1}^{2}-\Psi \Gamma_{2}
\end{aligned}
$$

where

$$
\Psi \triangleq \Psi_{1} \Psi_{2}
$$

Let $\left\{p_{i}\right\}_{i=1}^{4}$ be the desired closed-loop pole positions and

$$
Q(s) \triangleq s^{4}+K_{1} s^{3}+K_{2} s^{2}+K_{3} s+K_{4}
$$

the corresponding polynomial with $\left\{p_{i}\right\}_{i=1}^{4}$ as its roots. Matching the coefficients of (26) with (28) gives

$$
\begin{aligned}
2 \sigma \omega+2 \xi_{1} w_{1} & =K_{1} \\
\omega^{2}+2 \sigma \omega 2 \xi_{1} w_{1}+w_{1}^{2} & =K_{2} \\
2 \sigma \omega w_{1}^{2}+2 \xi_{1} w_{1} \omega^{2}-\Psi \Gamma_{1} & =K_{3} \\
\omega^{2} w_{1}^{2}-\Psi \Gamma_{2} & =K_{4} .
\end{aligned}
$$

Equations (29)-(32) imply

$$
\begin{aligned}
2 \xi_{1} w_{1} & =K_{1}-2 \sigma \omega \\
w_{1}^{2} & =K_{2}-\omega^{2}-2 \sigma \omega 2 \xi_{1} w_{1} \\
& =K_{2}-\omega^{2}-2 \sigma \omega\left(K_{1}-2 \sigma \omega\right) \\
\Gamma_{1} & =\frac{1}{\Psi}\left[w_{1}^{2} 2 \sigma \omega+\omega^{2} 2 \xi_{1} w_{1}-K_{3}\right] \\
\Gamma_{2} & =\frac{1}{\Psi}\left[w_{1}^{2} \omega^{2}-K_{4}\right] .
\end{aligned}
$$

In other words, using the PVPF controller (11) one could place the poles of the closed-loop system (22) at any desired location. However, as

$$
\begin{aligned}
2 \xi_{1} w_{1} & =2 \xi w-\Gamma_{1} d \\
w_{1}^{2} & =w^{2}-\Gamma_{2} d
\end{aligned}
$$

the choice of $K_{1}, K_{2}, K_{3}$, and $K_{4}$, or alternatively the pole positions $\left\{p_{i}\right\}_{i=1}^{4}$, should not be such that the controller damping

$$
\left[\begin{array}{c}
\ddot{x} \\
\ddot{z}
\end{array}\right]+\left[\begin{array}{cc}
2 \sigma \omega & 0 \\
0 & 2 \xi_{1} w_{1}
\end{array}\right]\left[\begin{array}{c}
\dot{x} \\
\dot{z}
\end{array}\right]+\left[\begin{array}{cc}
\omega^{2} & 0 \\
0 & w_{1}^{2}
\end{array}\right]\left[\begin{array}{c}
x \\
z
\end{array}\right]=\left[\begin{array}{cc}
0 & 0 \\
\Gamma_{1} \Psi_{2} & 0
\end{array}\right]\left[\begin{array}{c}
\dot{x} \\
\dot{z}
\end{array}\right]+\left[\begin{array}{cc}
0 & \Psi_{1} \\
\Gamma_{2} \Psi_{2} & 0
\end{array}\right]\left[\begin{array}{c}
x \\
z
\end{array}\right]+\left[\begin{array}{c}
0 \\
\Gamma_{2} d
\end{array}\right] \dot{r}(t)+\left[\begin{array}{c}
\Psi_{1} \\
\Gamma_{2} d
\end{array}\right] r(t)
$$


$2 \xi w$ and the square of the controller frequency $w^{2}$ become negative. Hence, the choices should be such that

$$
\begin{aligned}
2 \xi w & =2 \xi_{1} w_{1}+\Gamma_{1} d>0 \\
w^{2} & =w_{1}^{2}+\Gamma_{2} d>0 .
\end{aligned}
$$

Using constraints (39) and (40), controller stability can be characterized in terms of linear inequalities

$$
\begin{array}{r}
a_{1} K_{1}+a_{2} K_{2}+a_{3} K_{3}>a_{4} \\
b_{1} K_{1}+b_{2} K_{2}+b_{3} K_{4}>b_{4}
\end{array}
$$

where

$$
\begin{aligned}
& a_{1}=\left(1-\frac{d}{\Psi}\left((2 \sigma \omega)^{2}-\omega^{2}\right)\right) \\
& a_{2}=2 \sigma \omega \frac{d}{\Psi} \\
& a_{3}=-\frac{d}{\Psi} \\
& a_{4}=2 \sigma \omega-\frac{d}{\Psi}\left((2 \sigma \omega)^{3}-4 \sigma \omega^{3}\right) \\
& b_{1}=-\left(2 \sigma \omega+\frac{d}{\Psi} 2 \sigma \omega^{3}\right) \\
& b_{2}=\left(1+\frac{d}{\Psi} \omega^{2}\right) \\
& b_{3}=-\frac{d}{\Psi} \\
& b_{4}=\left[\omega^{2}-(2 \sigma \omega)^{2}+\frac{d}{\Psi}\left(\omega^{4}-(2 \sigma \omega)^{2} \omega^{2}\right)\right] .
\end{aligned}
$$

\section{NUMERICAL ILLUSTRATIONS AND EXPERIMENTS USING PVPF}

In this section, a PVPF controller connecting the $V_{x^{-}}$output to the input $V_{x^{+}}$is constructed to damp the resonance in the transfer function $G_{x x}^{(v)}(s)$.

Poles of $G_{x x}^{(v)}(s)$, computed from (5), are

$$
p_{ \pm}=-30.1 \pm i 5337.3 \text {. }
$$

Here, the desired closed-loop poles are set to

$$
\begin{aligned}
& P_{1+}=P_{2+}=-2030.1 \pm i 5337.3 \\
& P_{1-}=P_{2-}=-2030.1 \pm i 5337.3 .
\end{aligned}
$$

In other words, the closed-loop poles of $G_{x x}^{(v)}(s)$ are further pushed into the left half plane by 2000 units.

It can be checked that the polynomial coefficients $K_{1}=$ $8.1204 \times 10^{3}, K_{2}=8.1701 \times 10^{7}, K_{3}=2.6479 \times 10^{11}$, and $K_{4}=1.0633 \times 10^{15}$ corresponding to the desired closed-loop poles $P_{1+}, P_{2+}, P_{1-}$, and $P_{2-}$, (52), satisfy the inequalities (41) and (42). Solving for the controller parameters $\Gamma_{1}, \Gamma_{2}, \xi$, and $\omega$ from (33) to (38), we obtain the PVPF controller

$$
K_{\mathrm{PVPF}}(s) \triangleq \frac{-1915 s+2.626 .10^{7}}{s^{2}+8582 s+4.558 .10^{7}}
$$

that would render a closed-loop system having poles at $P_{1+}$, $P_{2+}, P_{1-}$, and $P_{2-}$. In Fig. 11 , the FRF $K_{\mathrm{PVPF}}(i \omega)$ of the
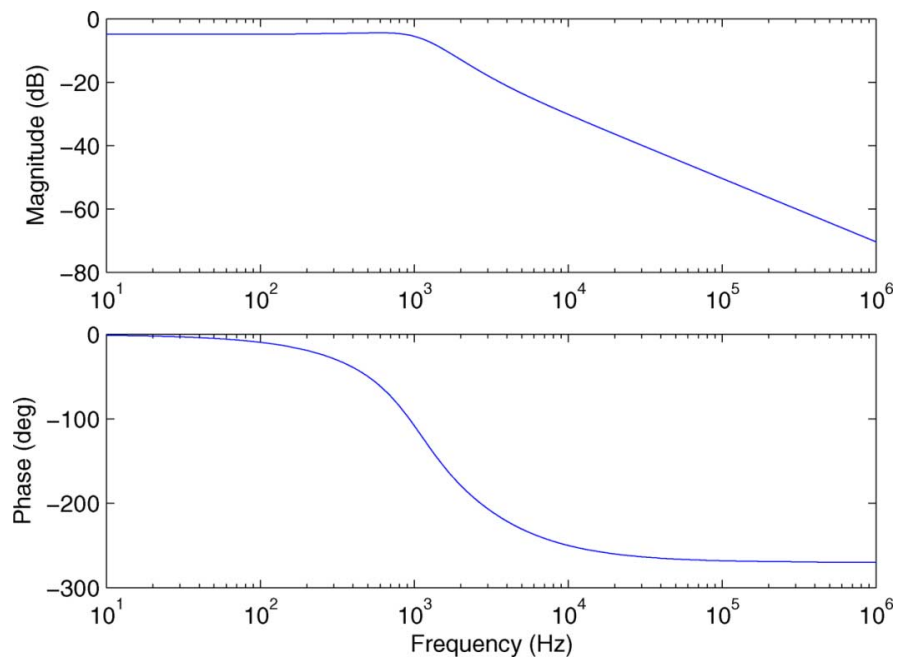

Fig. 11. Bode plot of the PVPF controller $K_{\mathrm{PVPF}}$.

controller is plotted. The low gain and the quick roll off of the FRF $K_{\mathrm{PVPF}}(i \omega)$ suggest that the control effort needed to push the real part of the poles $p_{ \pm}$(51) to $P_{1+}, P_{2+}, P_{1-}$, and $P_{2-}$ is not very high. Hence, the controller $K_{\mathrm{PVPF}}(s)$ is very easily implementable.

A dSPACE DS1103 rapid prototyping system is used for the real-time implementation of the controller. Simulink and MATLAB are used to download the controller (53) into the board. The sampling frequency of the controller board is set to $20 \mathrm{kHz}$ in order to avoid aliasing.

In what follows, the effectiveness of the PVPF controller in damping the resonance of $G_{x x}^{(v)}(s)$ will be evaluated both numerically and experimentally. Evaluating numerically refers to using the expressions obtained for $\Gamma_{1}, \Gamma_{2}, \xi$, and $\omega$ in the closedloop model (24) and comparing it with the open-loop model $G_{x x}^{(v)}(s)(5)$. While evaluating experimentally refers to applying swept sine waves to the $x^{+}$electrode of the tube and determining the FRFs of the closed-loop system and comparing them with their respective open-loop counterparts. Furthermore, the effect of the PVPF controller on the transfer functions $G_{d_{x} x}^{(v)}$ and $G_{d_{y} x}^{(v)}$ of the subsystem $C$ is also examined.

In Fig. 12, the frequency response of the closed-loop model (24) is plotted, along with the open-loop model (5). A damping of around $30 \mathrm{~dB}$ at the resonance is evident from the plot. In Fig. 13, the Frequency response of the closed-loop system FRFs obtained experimentally is plotted along with the corresponding open-loop FRFs in $G_{v v}(i \omega)$. It is evident from Figs. 12 and 13 that in the case of $G_{x x}^{(v)}(s)$ the damping predicted numerically is matched by the experimental results. Furthermore, experimental results suggest a significant damping of the resonance in the FRF of the cross coupling term $G_{x y}^{(v)}(s)$. As in the case of $G_{x x}^{(v)}(s)$ the effect of the feedback on $G_{x y}^{(v)}(s)$ can be mathematically characterized. However, that characterization is not presented here as it is neither important nor difficult.

In order to actuate the tube in a raster pattern, a triangular waveform with amplitude of $30 \mathrm{~V}$ and fundamental frequency of $40 \mathrm{~Hz}$ is applied to the closed-loop system. In Fig. 14, 


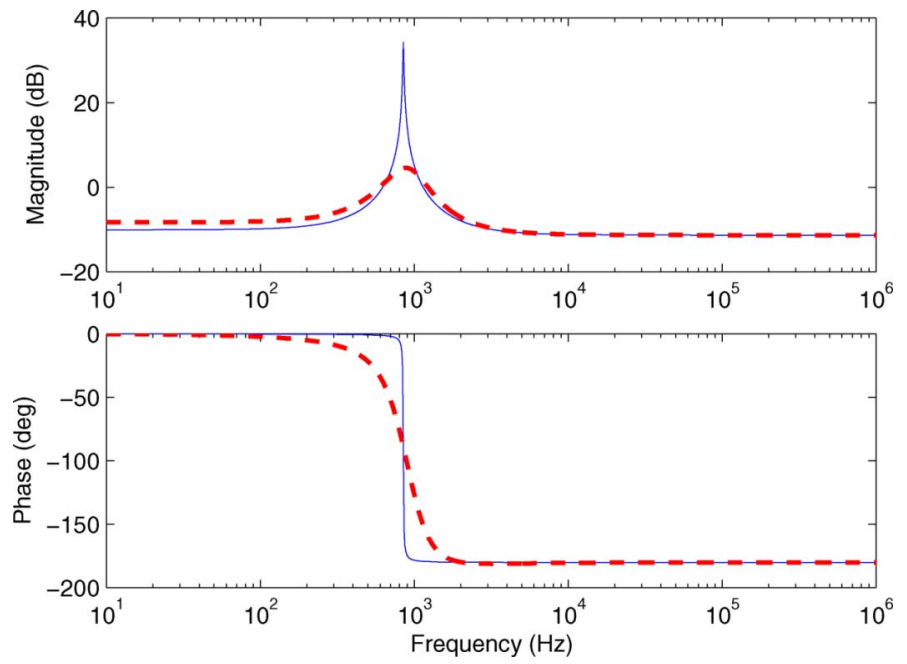

Fig. 12. Frequency response of the open-loop model (solid line) (5) along with the closed-loop model (dotted line) (24).
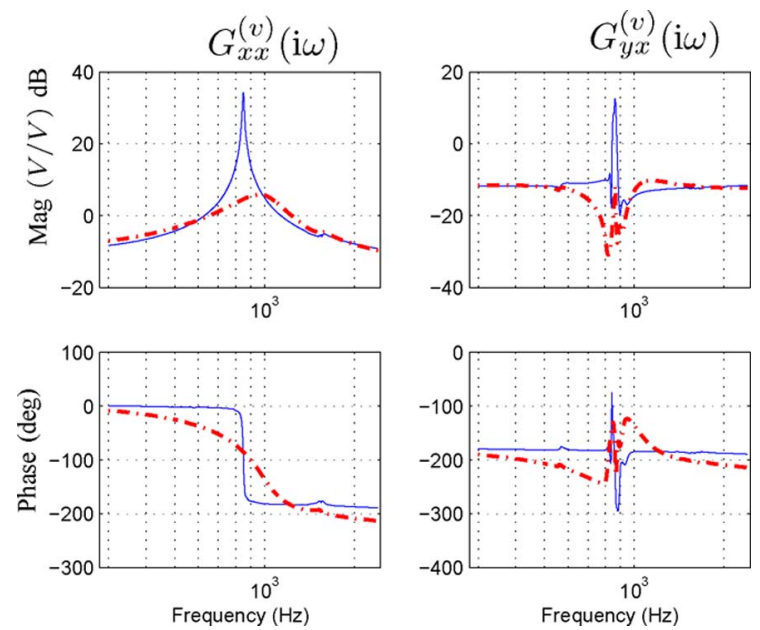

Fig. 13. Closed-loop response of the subsystem $V$ (dashed dotted line) along with its open loop counterpart (solid line).

the capacitive sensor output $d_{x}$ in response to the triangular waveform input is plotted. It is apparent that the periodic corrugations in open-loop capacitive sensor response are not present in the corresponding closed-loop response. Nevertheless, the closed-loop capacitive sensor response is not really a smooth triangular waveform, in particular, near the peaks of the response. In Fig. 15, the closed-loop frequency responses (FRFs) of the capacitive sensors are plotted. Note that, as in the case of the closed-loop frequency responses of the voltage subsystem, the closed-loop frequency responses of the capacitive subsystem also have their resonances damped. As a consequence, harmonics of the triangular waveform close to the resonance are not amplified. However, to obtain a smooth triangular waveform at the output, as in (7), an input

$$
r(t) \triangleq \sum_{k=1}^{\infty} \frac{a_{k}}{\left|G_{d_{x} x}^{v,(c l)}\left(i \omega_{k}\right)\right|} \sin \left(\omega_{k} t-\phi_{k}\right)
$$

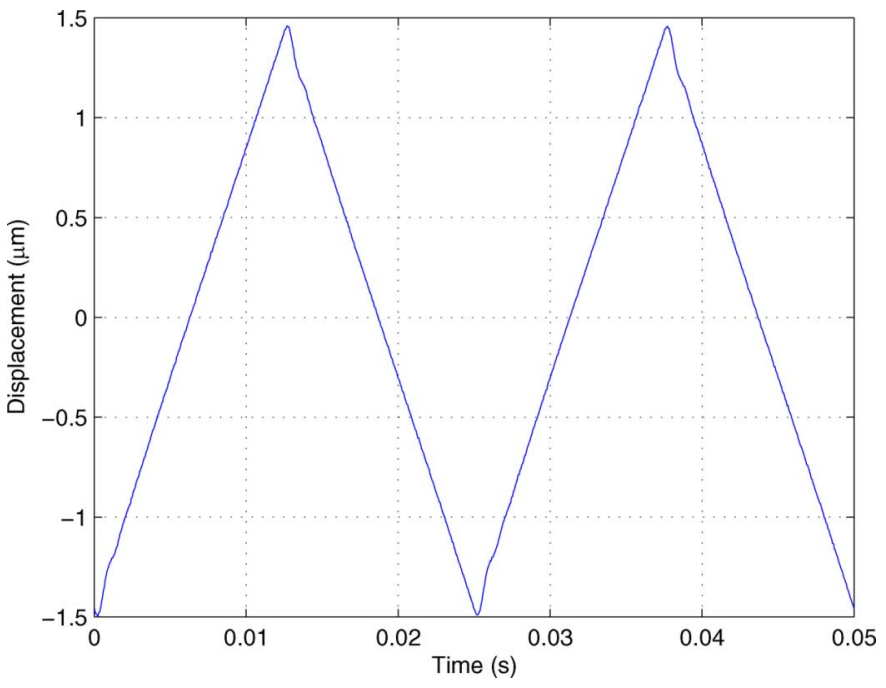

Fig. 14. Response recorded by the capacitive sensor $d_{x}$ for a triangular waveform input with an amplitude of $30 \mathrm{~V}$ and a fundamental frequency $40 \mathrm{~Hz}$.
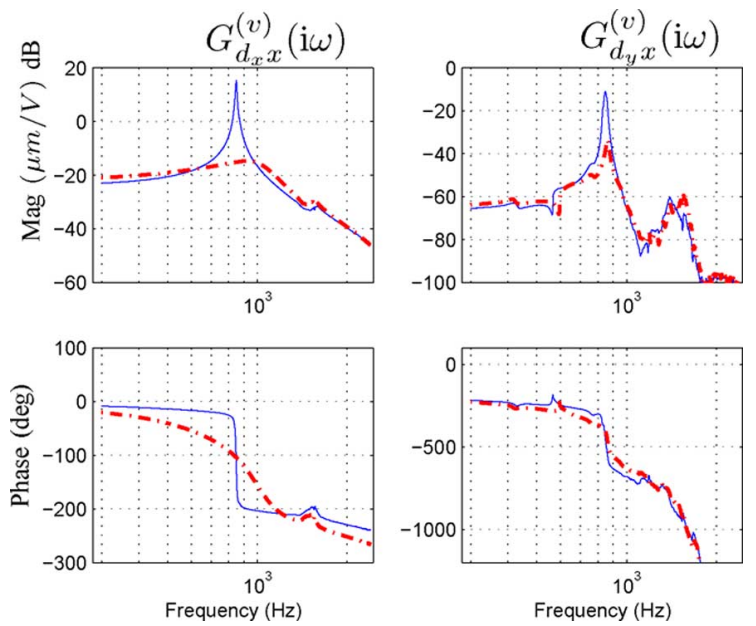

Fig. 15. Closed-loop response of the subsystem $C$ (dashed dotted line) along with its open-loop counterpart (solid line).

is designed. In (54), $a_{k}$ and $\omega_{k}$ are the Fourier components of the desired triangular waveform, and $G_{d_{x} x}^{(v, c l)}(i \omega)$ denotes the FRF of the transfer function $G_{d_{x} x}^{(v, c l)}(s)$ fitted for the closed-loop data plotted in Fig. 15 and $\phi_{k}=\arg G_{d_{x} x}^{v,(c l)}\left(i \omega_{k}\right)$. The response $d_{x}$ to such an input is shown in Fig. 16, which is a smooth triangular waveform.

Finally, to illustrate the robustness introduced into the system by the PVPF controller, as before, the system resonance frequency is perturbed by placing a nonconducting mass on the bonded aluminum cube. The same PVPF controller $K_{\mathrm{PVPF}}(53)$ and the filtered input $r(t)$ (54) are used. In Fig. 17, the response $d_{x}$ of the perturbed closed-loop system to the filtered input $r(t)$ is plotted. It can be noted that the output is still a triangular 


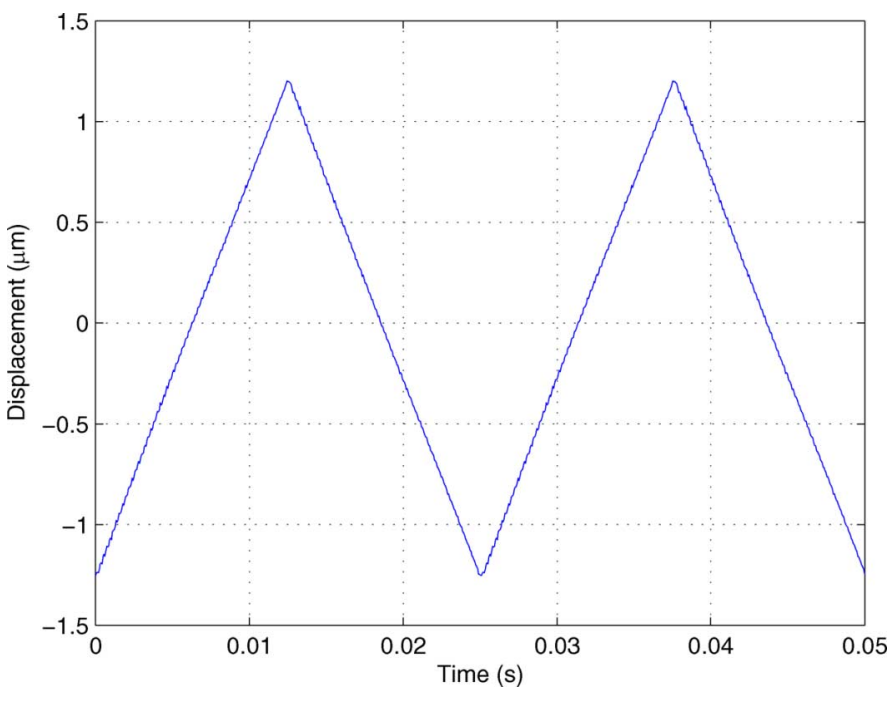

Fig. 16. Response recorded by the capacitive sensor $d_{x}$ for an input of the form (54) with the triangular waveform having an amplitude of $30 \mathrm{~V}$ and a fundamental frequency of $40 \mathrm{~Hz}$.

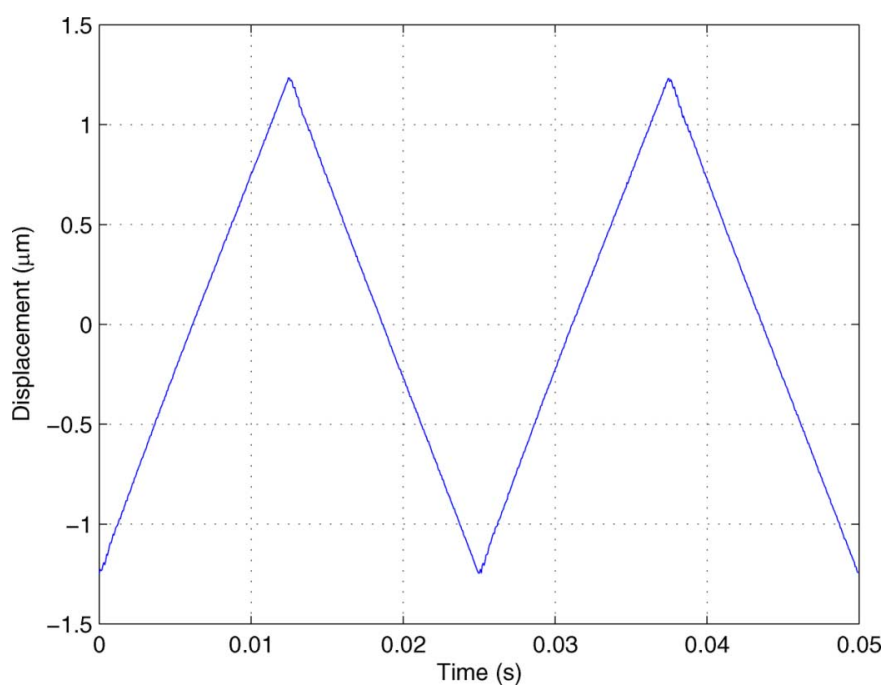

Fig. 17. Response recorded by the capacitive sensor $d_{x}$ for an input of the form (54) with the triangular waveform having an amplitude of $30 \mathrm{~V}$ and a fundamental frequency of $40 \mathrm{~Hz}$ when the system resonance is perturbed.

waveform similar to the one in Fig. 16. Thus confirming the robustness introduced into the system by the PVPF controller.

\section{DEALING With HySTERESIS}

So far in this paper, hysteresis has not been encountered. No hysteresis is visible in Figs. 16 and 17. It is well known that piezoelectric tubes and actuators display significant hysteresis [23], [26]-[30] when excited with high amplitude voltage signals. In the current context, hysteresis becomes evident when the input amplitude is increased from 30 to $32 \mathrm{~V}$. The most common way to illustrate the presence of hysteresis is to plot input versus output directly (see [19], [23], [28], and [29]). In Fig. 18, the capacitive sensor response $d_{x}$, in closed loop, to a sine wave input with amplitude $32 \mathrm{~V}$ and frequency $40 \mathrm{~Hz}$ is plotted. It is clear from Fig. 18 that the output $d_{x}$ recorded is not a pure sine wave, as the $\mathrm{I} / \mathrm{O}$ plot is nonlinear. Since the capacitive sensor response

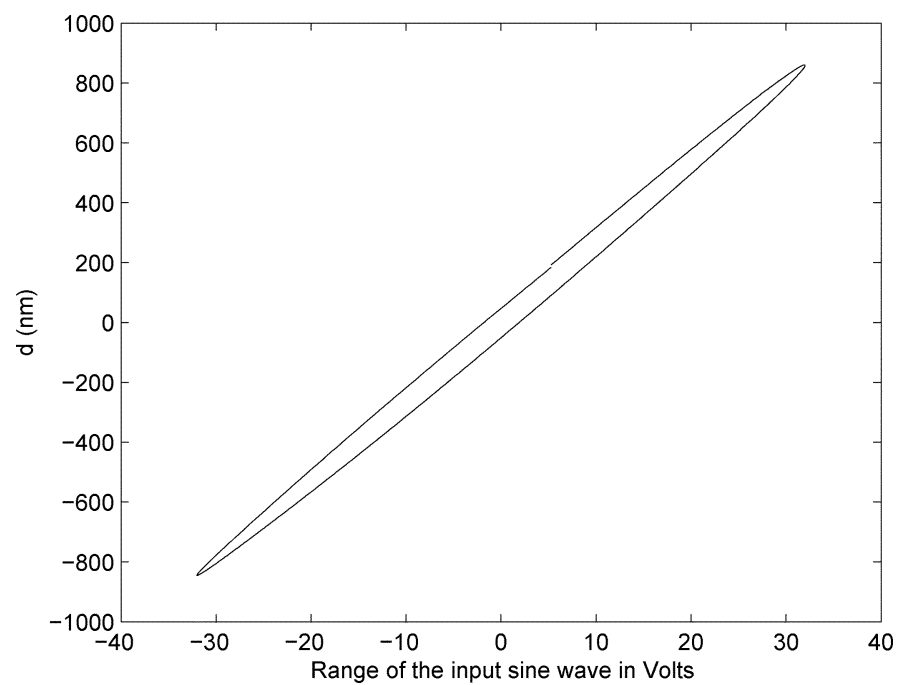

Fig. 18. Response recorded by the capacitive sensor $d_{x}$ to a sine wave input with amplitude $32 \mathrm{~V}$ and frequency $40 \mathrm{~Hz}$.

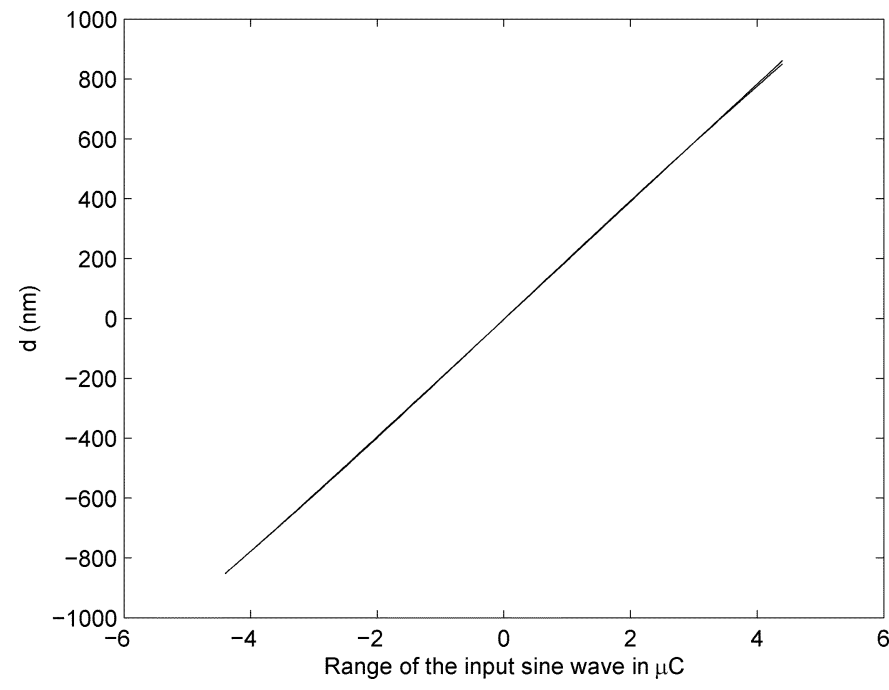

Fig. 19. Response recorded by the capacitive sensor $C_{x}$ to a sine wave input with amplitude $4.4 \mu \mathrm{C}$ and frequency $40 \mathrm{~Hz}$.

was recorded in the closed loop it is also clear that feedback control alone is not sufficient to suppress hysteresis.

As mentioned earlier, it has been known since the late 1980s that driving piezoelectric transducers with current or charge rather than voltage significantly reduces hysteresis. In Fig. 19, the response recorded by the capacitive sensor $d_{x}$, in closed loop, to a sine wave input with amplitude $4.4 \mu \mathrm{C}$ and frequency $40 \mathrm{~Hz}$ is plotted. Note that the amplitude of the capacitive sensor response is similar to that obtained for the voltage case, and more importantly, the hysteresis observed in Fig. 18 is more or less absent in this case. In spite of the benefits, charge actuation has not been generally accepted due to the practical problems of voltage drift and poor low-frequency response [23], [25] of the charge sources, problems that are seldom encountered in voltage amplifiers. In the following subsections, details on the design of the charge amplifier and its effect on the dynamics of the tube are presented. The information presented in these subsections can also be found in [28] in more detail. 


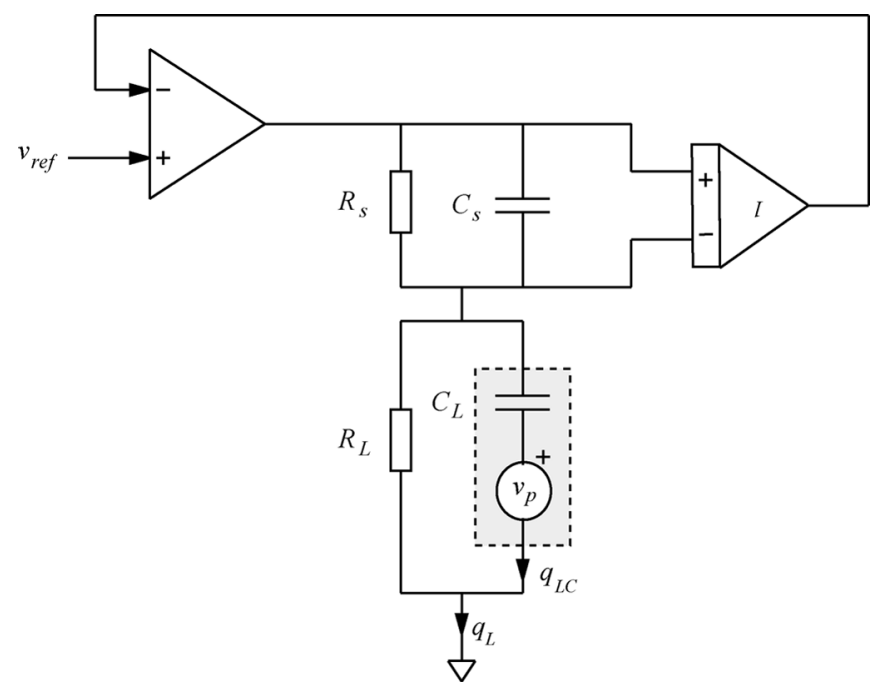

Fig. 20. DC accurate charge source for grounded capacitive loads.

Nevertheless, for the sake of completion and also to maintain the continuity of the paper, for the benefit of the reader, we present some of these details.

\section{A. Linearization By Charge Drive}

Although the circuit topology of a charge or current amplifier is much the same as a simple voltage amplifier, the uncontrolled nature of the output voltage typically results in the load capacitor being charged up. Saturation and distortion occur when the output voltage, referred to as the compliance voltage, reaches the power supply rails.

A solution for the problem of voltage drift was first presented in [31]. An auxiliary voltage feedback loop was included to correct low-frequency behavior and allow for constant charge offsets. The circuit implementation required the design of separate voltage and charge feedback controllers. A simplified design relying on the intrinsic voltage control offered by the parasitic resistances was later presented in [32]. A later extension allowing for grounded loads was presented in [33]. As piezoelectric tubes have multiple external electrodes and a common (often grounded) internal electrode, the requirement for a grounded-load is a necessity.

The simplified schematic of a dc-accurate grounded-load charge amplifier is shown in Fig. 20. The piezoelectric transducer, shaded in gray, is grounded, while the charge sensing capacitor $C_{s}$, is floating. The amplifier works to equate the voltage measured across the sensing impedance to the reference voltage $v_{\text {ref }}$.

By setting $C_{L} R_{L}=C_{s} R_{s}$, i.e.,

$$
\frac{R_{L}}{R_{s}}=\frac{C_{s}}{C_{L}}
$$

it can be shown that the amplifier has a constant gain $C_{s} \mathrm{C} / \mathrm{V}$ over all frequencies, including dc [33]. The voltage amplifier formed by the two resistances $R_{L}$ and $R_{s}$ effectively synthesizes the operation of an ideal charge amplifier at low frequencies. At frequencies above $f_{c}=1 / R_{L} C_{L} \mathrm{~Hz}$, the amplifier is charge dominant and voltage dominant below [33].
Similar to a typical voltage amplifier, the amplifier offers little or no hysteresis reduction over the frequency range of voltage dominance.

\section{B. Relationship Between Voltage and Charge Actuation}

In the previous sections, modeling of a piezoelectric tube scanner was performed using data obtained from a voltage driven system. Due to the benefits in reducing hysteresis, only charge actuation will be considered in the proceeding sections. At this point, it is informative to consider the relationship between the two actuation mechanisms: voltage and charge.

Consider the electrically equivalent model of a voltage and charge driven piezoelectric tube shown in Fig. 21. Each electrode acts as a piezoelectric transducer, represented by a strain dependent voltage source $v_{p}$ and a series capacitor $C_{p}$. The polarization vector is assumed to be oriented radially outward. In this case, a positive voltage or charge results in a positive deflection. Previously, we have been interested in the transfer functions from an applied voltage $v$ to the resulting piezoelectric voltage $v_{p}$ and tip translation $d$, that is

$$
G_{v v}(s)=\frac{v_{p}(s)}{v(s)} \quad G_{d v}(s)=\frac{d(s)}{v(s)} .
$$

The transfer functions $G_{v v}(s)$ and $G_{d v}(s)$ can be derived analytically or experimentally as performed in Section II.

In the case of charge actuation [see Fig. 21(b)], equivalent transfer functions can be derived. Kirchoff's voltage law for the loop is

$$
\frac{-q}{C_{p}}+v_{p}+v_{q}=0
$$

Substituting $v_{p}=G_{v v} v_{q}$ and simplifying yields the relationship between $G_{v v}$ and the charge driven equivalent $G_{v q}$

$$
G_{v q}(s)=\frac{v_{p}(s)}{q(s)}=\frac{1}{C_{p}} \frac{G_{v v}(s)}{1+G_{v v}(s)} .
$$

The displacement transfer function can be derived in a similar fashion

$$
G_{d q}(s)=\frac{d(s)}{q(s)}=\frac{1}{C_{p}} \frac{G_{d v}(s)}{1+G_{v v}(s)} .
$$

Off resonance, where $G_{v v}(s) \ll 1$

$$
G_{v q}(s) \approx \frac{G_{v v}(s)}{C_{p}} \quad G_{d q}(s) \approx \frac{G_{d v}(s)}{C_{p}} .
$$

Thus, the relationship between charge and voltage actuation is revealed. Off resonance, the two driving mechanisms are related approximately by a constant. Due to the unity feedback of $G_{v v}$ in $G_{v q}$, the tube appears slightly "stiffer" with slightly higher resonance frequencies. This effect is due physically to the high AC impedance of a charge source.

\section{System Identification: Charge}

In this subsection, the dynamics of the piezoelectric tube are identified using charge signals. In other words, using the experimental setup described in Section II, the FRFs

$$
G_{v q}(i \omega) \triangleq\left[\begin{array}{ll}
G_{x x}^{(q)}(i \omega) & G_{x y}^{(q)}(i \omega) \\
G_{y x}^{(q)}(i \omega) & G_{y y}^{(q)}(i \omega)
\end{array}\right]
$$




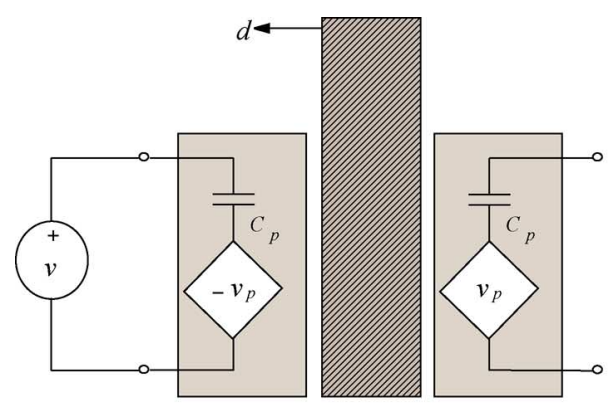

(a)

Fig. 21. (a) Voltage and (b) charge driven piezoelectric tube.
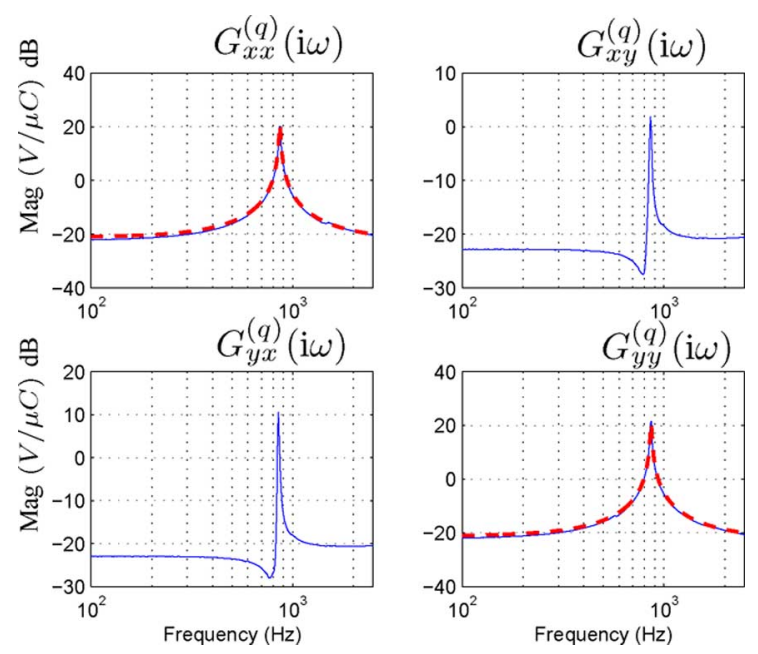

Fig. 22. Magnitude plots of the data (solid line) along with their corresponding parametric models (dashed dotted line).

$$
G_{d q}(i \omega) \triangleq\left[\begin{array}{ll}
G_{d_{x} x}^{(q)}(i \omega) & G_{d_{y} y}^{(q)}(i \omega) \\
G_{d_{x} y}^{(q)}(i \omega) & G_{d_{y} y}^{(q)}(i \omega)
\end{array}\right]
$$

are identified. Here, both the subscript and the superscript $q$ are used to denote the fact that inputs are charge signals $\left[Q_{x^{+}}, Q_{y^{+}}\right]^{\top}$ as opposed to voltage signals $\left[V_{x^{+}}, V_{y^{+}}\right]^{\top}$ used in Section II.

The FRFs $G_{v q}(i \omega)$ and $G_{d q}(i \omega)$ are determined by applying swept sine waves of charge signals into the electrodes $x^{+}$and $y^{+}$, and recording the corresponding voltage outputs at $x^{-}$ and $y^{-}$and the capacitive sensor outputs $d_{x}$ and $d_{y}$ using the same HP 35670A dual channel Spectrum Analyzer. In Fig. 22, the magnitude of the FRFs $G_{x x}^{(q)}(i \omega), G_{y y}^{(q)}(i \omega), G_{x y}^{(q)}(i \omega)$, and $G_{y x}^{(q)}(i \omega)$, are plotted. Similarly, in Fig. 23, the magnitude of the FRFs $G_{d_{x} x}^{(q)}(i \omega), G_{d_{y} x}^{(q)}(i \omega), G_{d_{x} y}^{(q)}(i \omega)$, and $G_{d_{y} y}^{(q)}(i \omega)$ are plotted.

As in the case of FRFs in Section II, it can be noted from the plots that $\left|G_{x x}^{(q)}(i \omega) \quad\right| \approx\left|G_{y y}^{(q)}(i \omega) \quad\right|$ and $\left|G_{d_{x} x}^{(q)}(i \omega) \quad\right| \approx \mid G_{d_{y} y}^{(q)}(i \omega) \quad$, but the magnitudes of the cross coupling terms differ from one another. As expected the cross coupling terms $G_{d_{y} x}^{(q)}(i \omega)$ and $G_{d_{x} y}^{(q)}(i \omega)$ in the subsystem $C$ are negligible in magnitude, except at the frequencies around

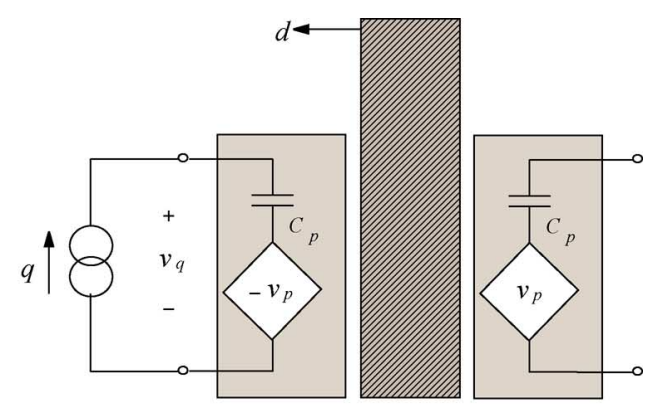

(b)
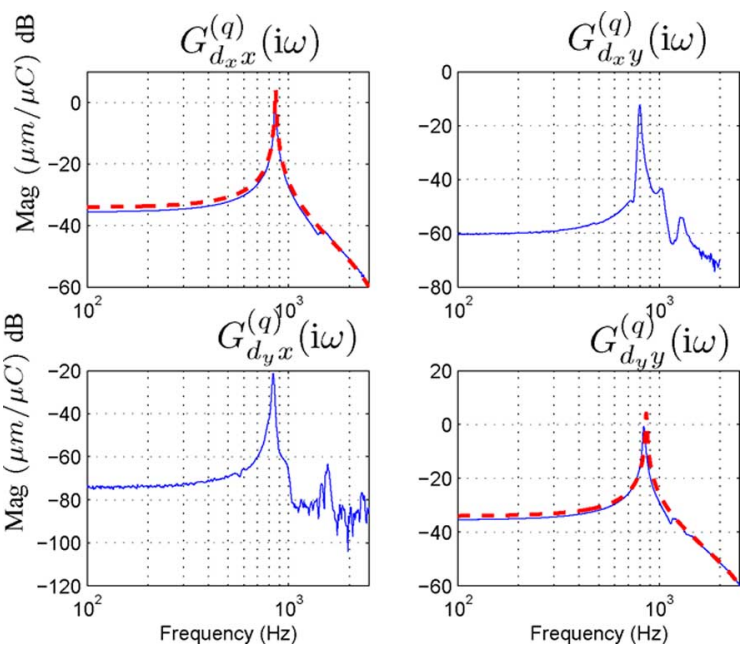

Fig. 23. Magnitude plots of the data (solid line) along with their corresponding parametric models (dashed dotted line).

TABLE II

PARAMETER VALUES OF THE FRFs $G_{d_{x} x}^{(q)}(s)$ AND $G_{d_{y} y}^{(q)}(s)$

\begin{tabular}{|c|c|}
\hline$k_{1}$ & $4.8753 e+006$ \\
\hline $2 \sigma \omega$ & 78.9826 \\
\hline$\omega^{2}$ & $2.9426 e+007$ \\
\hline$d_{1}$ & $-7.8 \times 10^{-2}$ \\
\hline$c_{1}$ & 0.0015 \\
\hline$c_{2}$ & 0.1185 \\
\hline$c_{3}$ & $5.874 \times 10^{5}$ \\
\hline
\end{tabular}

the resonance, when compared with the direct terms $G_{d_{x} x}^{(q)}(i \omega)$ and $G_{d_{y} y}^{(q)}(i \omega)$ (see Fig. 23).

\section{Modeling}

Second-order models are fit to the data. The following models were found to be good fits:

$$
\begin{aligned}
G_{x x}^{(q)}(s) & =(q)_{y y}(s)=\frac{k_{1}}{s^{2}+2 \sigma \omega s+\omega^{2}}+d_{1} \\
G_{d_{x} x}^{(q)}(s) & =G_{d_{y} y}^{(q)}(s)=\frac{c_{1} s^{2}+c_{2} s+c_{3}}{s^{2}+2 \sigma \omega s+\omega^{2}} .
\end{aligned}
$$

The model parameters are as tabulated in Table II. It can be seen from Figs. 22 and 23 that the parametric models (63) and (64) fit their respective data reasonably well. 

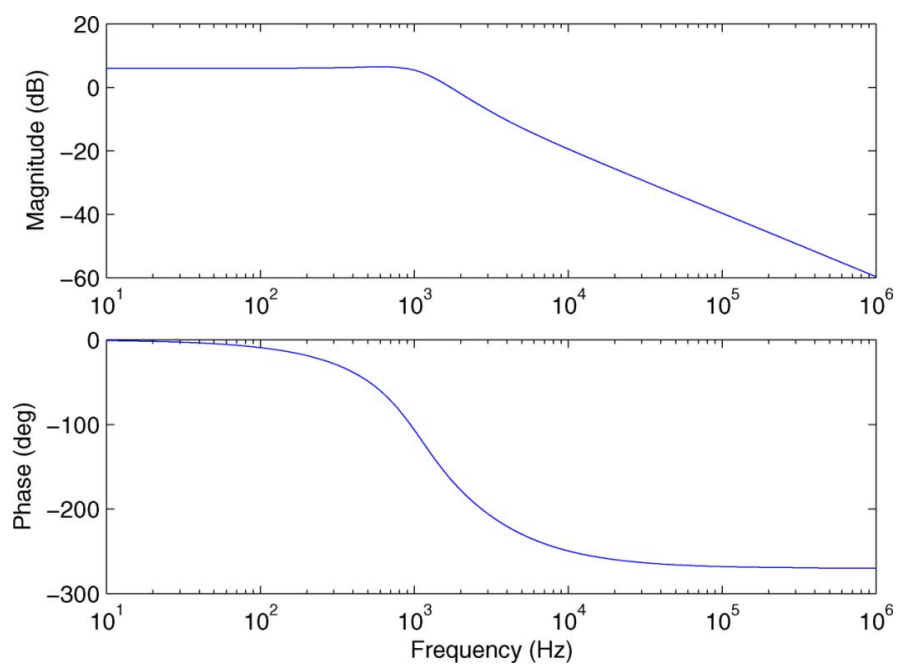

Fig. 24. Bode plot of the PVPF controller $K_{\mathrm{QPVPF}}$.

\section{E. PVPF Control: Charge}

In this subsection, a PVPF controller is constructed to damp the resonance in the transfer function $G_{x x}^{(q)}(s)$. Note that the poles of $G_{x x}^{(q)}(s)$, computed from (63) are

$$
p_{ \pm}=-39.5 \pm i 5424.4
$$

As in Section V, it is desired to push the closed-loop poles of $G_{x x}^{(q)}(s)$ further into left half plane by 2000 units. In other words, the desired closed-loop poles are

$$
\begin{aligned}
& P_{1+}=P_{2+}=-2039.5 \pm i 5424.4 \\
& P_{1-}=P_{2-}=-2039.5 \pm i 5424.4 .
\end{aligned}
$$

It can be checked that the polynomial coefficients $K_{1}=$ $8.158 \times 10^{3}, K_{2}=8.381 \times 10^{7}, K_{3}=2.74 \times 10^{11}$, and $K_{4}=1.128 \times 10^{15}$ corresponding to the desired closed-loop poles $P_{1+}, P_{2+}, P_{1-}$, and $P_{2-}$ (66), satisfy the inequalities (41) and (42). Solving for the controller parameters $\Gamma_{1}, \Gamma_{2}, \xi$, and $\omega$ from (33)-(38), the PVPF controller

$$
K_{\mathrm{QPVPF}}(s) \triangleq \frac{-6564 s+9.302 .10^{7}}{s^{2}+8591 s+4.649 .10^{7}}
$$

that would render a closed-loop system having poles at $P_{1+}, P_{2+}, P_{1-}$, and $P_{2-}$ is obtained. In Fig. 24, the FRF $K_{\mathrm{QPVPF}}(i \omega)$ is plotted. It is worth noting that the controller $K_{\mathrm{QPVPF}}(i \omega)$ is rather similar to the controller $K_{\mathrm{PVPF}}(i \omega)$ plotted in Fig. 11.

In the remainder of this subsection, the effectiveness of the controller $K_{\mathrm{QPVPF}}(i \omega)$ in damping the resonant peaks of the FRFs $G_{x x}^{(q)}(s), G_{y y}^{(q)}(s), G_{d_{x} x}^{(q)}$, and $G_{d_{y} x}^{(q)}$ will be examined both numerically and experimentally. In Fig. 25, the frequency response of the closed-loop model is plotted, along with the openloop model (56). A damping of around $30 \mathrm{~dB}$ at the resonance is evident from the plot. In Fig. 26, the frequency response of the closed-loop system FRFs obtained experimentally is plotted along with the corresponding experimentally determined open-
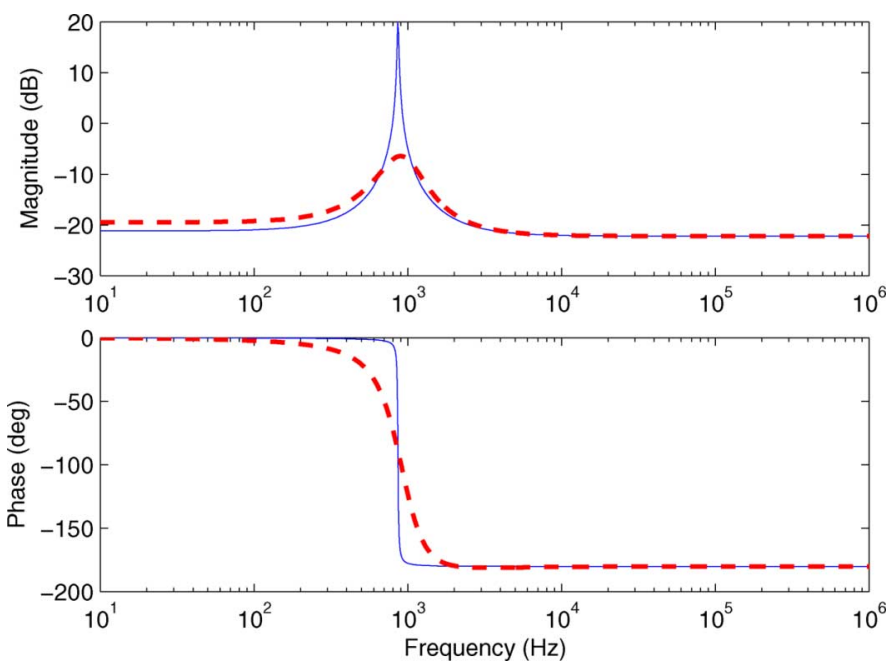

Fig. 25. Frequency response of the closed-loop model (dotted line) along with the open-loop model (solid line) (63).
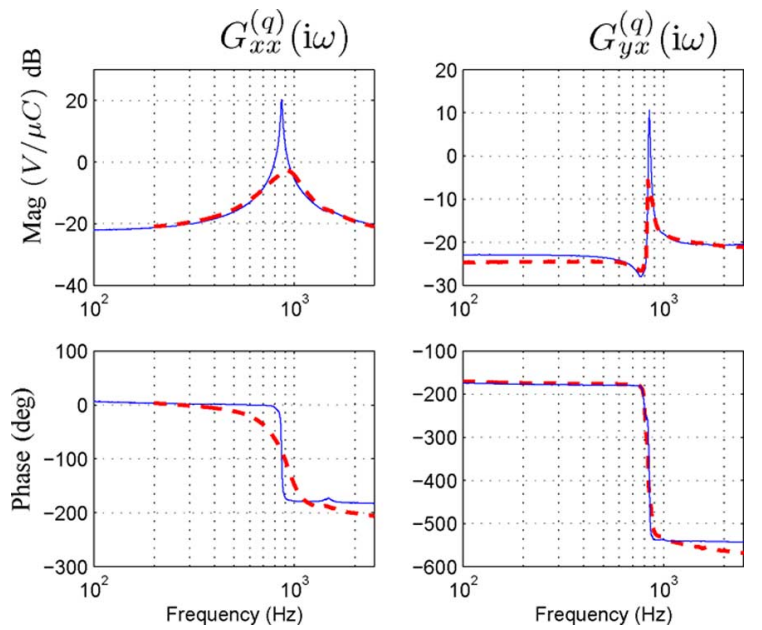

Fig. 26. Closed-loop response of the subsystem $V$ (dashed dotted line) along with its open-loop counterpart (solid line).

loop FRFs in $G_{v q}(i \omega)$. It is evident from Figs. 25 and 26 that in the case of $G_{x x}^{(q)}(s)$ the damping predicted numerically is matched by the experimental results presented. Furthermore, as observed in Section V, experimental results suggest a massive damping of the resonance in the cross coupling term $G_{x y}^{(q)}(s)$. In Fig. 27, the frequency response of the closed-loop system FRFs obtained experimentally is plotted along with the respective open-loop FRFs in $G_{d q}(i \omega)$. As in the case of voltage subsystem, a good damping of the capacitive sensor responses can also be observed.

As done in Section V, an input of the form

$$
r(t) \triangleq \sum_{k=1}^{\infty} \frac{a_{k}}{\left|G_{d_{x} x}^{(q, c l)}\left(i \omega_{k}\right)\right|} \sin \left(\omega_{k} t-\phi_{k}\right)
$$

is designed. In (68), $a_{k}$ and $\omega_{k}$ are the Fourier components of the desired triangular waveform and $G_{d_{x} x}^{(q, c l)}(i \omega)$ denotes the FRF of the transfer function $G_{d_{x} x}^{(q, c l)}(s)$ fitted for the closed-loop data plotted in Fig. 27 and $\phi_{k}=\angle G_{d_{x} x}^{(q, c l)}\left(i \omega_{k}\right)$. The response 

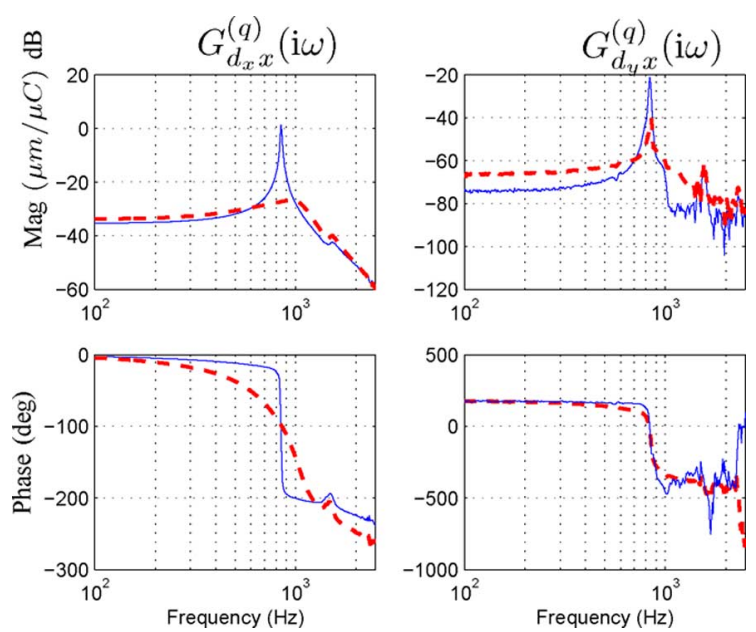

Fig. 27. Closed-loop response of the subsystem $C$ (dashed dotted line) along with its open-loop counterpart (solid line).

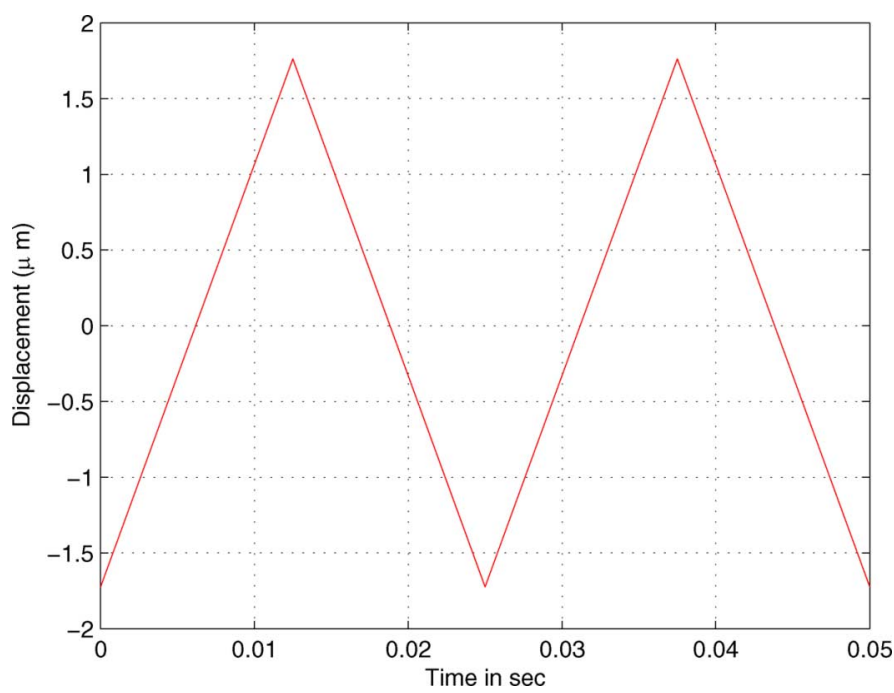

Fig. 28. Response recorded by the capacitive sensor $d_{x}$, for an input $r(t)$ of the form (68) with $f_{d}(t)$ being a triangular waveform with amplitude $5.5 \mu \mathrm{C}$ and fundamental frequency $40 \mathrm{~Hz}$.

recorded by the capacitive sensor $d_{x}$ to such an input $r(t)$ is plotted in Fig. 28.

\section{CONCLUSION}

In this paper, a piezoelectric tube scanner of the type typically used in STMs and AFMs is considered. The aim of this paper is to actuate the piezoelectric tube scanner in a raster pattern. Actuation of this tube is hampered by the presence of a low frequency resonance mode and hysteresis. The effect of hysteresis is significant only when actuating the piezoelectric tube at high amplitudes. At low amplitude actuations, only the effect of the resonant mode needs to be compensated.

In the case of low amplitude actuations, the piezoelectric tube is modeled as a linear system, and the lightly damped resonant mode hampering the actuation of the tube is identified. The concept of PVPF control is introduced, and a design procedure to damp the resonant mode using a PVPF controller is presented. It is observed that using a PVPF controller, a 30-dB damping of the resonant mode can be achieved without much control effort Inputs are shaped based on the closed-loop system to accurately actuate the piezoelectric tube in a raster pattern. It is also noted that the PVPF controller is robust to perturbations in the system parameters.

In the case of high amplitude actuations, where the hysteresis is prominent, the piezoelectric tube is actuated using a "homemade" charge amplifier. It is has been known for quite some time that actuation of piezoelectric materials using charge sources reduces hysteresis significantly (see [25] and [29]). However, traditionally, voltage amplifiers have been used to actuate piezoelectric materials as they are readily available. The use of a charge amplifier for actuation of piezoelectric tubes significantly reduces hysteresis and allows for modeling of the tube as a linear system. As in the low amplitude case, where voltage amplifiers were used, a PVPF controller is designed for the piezoelectric tube. Here too, a 30-dB damping of the resonant mode is achieved without much control effort. Inputs are then shaped based on the closed-loop system to accurately actuate the piezoelectric tube in a high amplitude raster pattern.

\section{REFERENCES}

[1] B. Bhushan, Ed., Springer Handbook of Nanotechnology. Heidelberg, Germany, Springer-Verlag, 2004.

[2] A. Daniele, S. Salapaka, M. V. Salapaka, and M. Daleh, "Piezoelectric tubes for atomic force microscopes: Design of lateral sensors, identification and control," in Proc. Amer. Control Conf., 1999, pp. 253-257.

[3] G. Schitter and A. Stemmer, "Identification and open-loop tracking control of a piezoelectric tube scanner for high-speed scanning probe microscopy," IEEE Trans. Control Syst. Technol., vol. 12, no. 3, pp. 449-454, May 2004.

[4] N. Tamer and M. Daleh, "Feedback control of piezoelectric tube scanners," in Proc. 33rd Conf. Dec. Control, 1994, pp. 1826-1831.

[5] O. M. El Rifai and K. Youcef-Toumi, "Coupling of piezoelectric tube scanning in scanning probe microscopes," in Proc. Amer. Control Conf., 2001, pp. 3251-3255.

[6] S. Salapaka and A. Sebastian, "Control of nanopositioning device," in Proc. 42nd IEEE Conf. Dec. Control, 2003, pp. 2644-2648.

[7] T. Ohara and K. Youcef-Toumi, "Dynamics and control of piezo tube actuators for subnanometer precision applications," in Proc. Amer. Control Conf., 1995, pp. 3808-3812.

[8] M. E. Taylor, "Dynamics of piezoelectric tube scanners for scanning probe microscopy," Rev. Sci. Instrum., vol. 64, no. 1, pp. 154-158, 1993.

[9] K. K. Leang and S. Devasia, "Hysteresis, creep and vibration compensation for piezoactuators: Feedback and feedforward control," in Proc. 2nd IFAC Conf. Mechatron. Syst., 2002, pp. 283-289.

[10] K. K. Leang and S. Devasia, "Iterative feedforward compensation of hysteresis in piezo positioners," in Proc. IEEE 42nd Conf. Dec. Control, 2003, pp. 2626-2631.

[11] J. L. Fanson and T. K. Caughey, "Positive position feedback control for large space structures," AIAA J., vol. 28, no. 4, pp. 717-724, 1990

[12] T. K. Caughey and C. J. Goh, "Analysis and control of quasi distributed parameter systems," California Inst. Technol., Pasadena, CA, Tech. Rep. DYNL-82-3, 1982.

[13] C. Choi and K. Park, "Self-sensing magnetic levitation using LC resonant circuit," Sens. Actuators, vol. 72, pp. 169-177, 1999.

[14] G. Song and B. N. Agrawal, "Vibration suppression of flexible spacecraft during attitude control," Acta Astronautica, vol. 49, no. 2, pp. 73-83, 2001.

[15] G. Song, S. Schmidt, and B. N. Agrawal, "Experimental study of vibration suppression of flexible spacecraft using modular control patch," in Proc. IEEE Aerosp. Conf., 1998, pp. 189-201.

[16] J. J. Dosch, D. J. Leo, and D. J. Inman, "Comparison of vibration control schemes for a smart antenna," in Proc. 31st Conf. Dec. Control, 1992, pp. 1815-1820.

[17] M. Ratnam, B. Bhikkaji, A. J. Fleming, and S. O. R. Moheimani, "PPF control of a piezoelectric tube scanner," in Proc. 44th IEEE Conf. Dec. Control Eur. Control Conf. (ECC), 2005, pp. 1168-1173. 
[18] S. O. R. Moheimani, B. J. G. Vautier, and B. Bhikkaji, "Experimental implementation of extended multivariable PPF control on an active structure," IEEE Trans. Control Syst. Technol., vol. 14, no. 3, pp. 443-445, May 2006.

[19] P. Ge and M. Jouaneh, "Tracking control of a piezoelectric actuator," IEEE Trans. Control Syst. Technol., vol. 4, no. 3, pp. 209-216, May 1996.

[20] D. Hughes and J. T. Wen, "Perisach modeling and compensation for smart material hysteresis," Proc. SPIE, Active Mater. Smart Structures, pp. 50-64, 1994.

[21] R. Venkataraman and P. S. Krishnaprasad, "Approximate inversion of hysteresis: Theory and numerical results," in Proc. 39th IEEE Conf. Dec. Control, 2000, pp. 4448-4454.

[22] X. Tan, R. Venkataraman, and P. S. Krishnaprasad, "Control of hysteresis: Theory and experimental results," Proc. SPIE, Model., Signal Process. Control, pp. 101-112, 2001.

[23] J. M. Cruz-Hayward and V. Hayward, "Phase control approach to hysteresis reduction," IEEE Trans. Control Syst. Technol., vol. 9, no. 1, pp. 17-26, Jan. 2001.

[24] C. V. Newcomb and I. Flinn, "Improving the linearity of piezoelectric ceramic actuators," Electron. Lett., vol. 18, no. 11, pp. 442-443, Nov. 1982.

[25] H. Kaizuka and B. Siu, "Simple way to reduce hysteresis and creep when using piezoelectric actuators," Jpn. J. Appl. Phys., Part 2-Lett., vol. 27, no. 5, pp. 773-776, May 1988.

[26] I. Mayergoyz, Mathematical Models of Hysteresis and their Applications. New York: Elsevier Science, 2003.

[27] A. J. Fleming and S. O. R. Moheimani, "Precision current and charge amplifiers for driving high capacitive piezoelectric loads," Electron. Lett., vol. 39, no. 3, pp. 282-284, Feb. 2003.

[28] A. J. Fleming and S. O. R. Moheimani, "Sensorless vibration supression and scan compensation for piezoelectric tube nanopositioners," IEEE Trans. Control Syst. Technol., vol. 14, no. 1, pp. 33-44, Jan. 2006.

[29] H. J. M. T. A. Adriaens, W. L. de Koning, and R. Banning, "Modeling piezoelectric actuators," IEEE/ASME Trans. Mechatron., vol. 5, no. 6, pp. 331-341, Dec. 2000.

[30] D. Croft, G. Shedd, and S. Devasia, "Creep, hysteresis and vibration compensation for piezoactuators: Atomic force microscopy application," in Proc. Amer. Control Conf., 2000, pp. 2123-2128.

[31] A. J. Fleming and S. O. R. Moheimani, "Hybrid DC accurate charge amplifier for linear piezoelectric positioning," in Proc. 3rd IFAC Symp. Mechatron. Syst., 2004, pp. 283-288.

[32] K. A. Yi and R. J. Veillette, "A charge controller for linear operation of a piezoelectric stack actuator," IEEE Trans. Control Syst. Technol., vol. 13 , no. 4, pp. 517-526, Jul. 2005.

[33] A. J. Fleming and S. O. R. Moheimani, "A grounded load charge amplifier for reducing hysteresis in piezoelectric tube scanners," Rev. Sci. Instrum., vol. 76, no. 7, pp. 22-44, 2005.

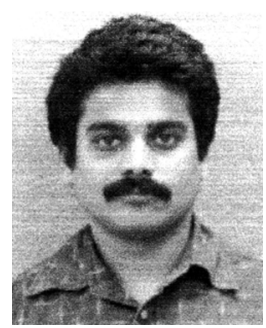

B. Bhikkaji received the Ph.D. degree in signal processing from the Uppsala University, Uppsala, Sweden, in 2004.

$\mathrm{He}$ is currently a Research Academic with the School of Electrical Engineering and Computer Science, The University of Newcastle, Callaghan, Australia. His research interests include system identification, robust control and active noise, and vibration control of flexible structures.

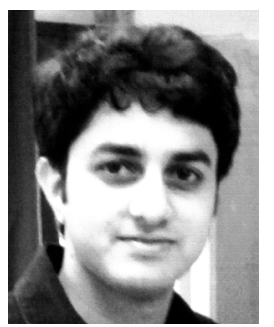

M. Ratnam received the B.Eng. degree in computer engineering and the B.Math. degree from the University of Newcastle, Callaghan, Australia, in 2003, where he is currently pursuing the M.Eng. degree in electrical engineering.

His research interests include control of piezoelectric tube scanners with a focus on increasing tracking bandwidth and resolution.

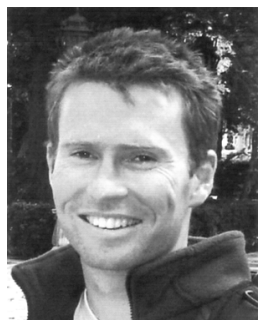

Andrew J. Fleming was born in Dingwall, U.K., in 1977. He received the B.Eng. and Ph.D. degrees in electrical engineering from the University of Newcastle, Callaghan, Australia, in 2000 and 2004, respectively.

$\mathrm{He}$ is currently an Australian Research Council Fellow stationed at the School of Electrical Engineering and Computer Science, University of Newcastle, where he was a Research Academic with the Center for Complex Dynamics and Control, University of Newcastle, in 2005. His research interests include micro-cantilever sensors, nano-positioning, and sensorless control of sound and vibration.

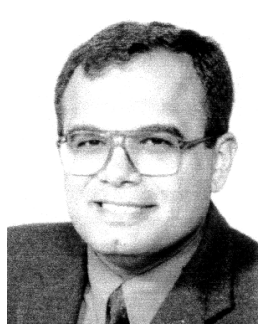

S. O. Reza Moheimani (SM'00) received the Ph.D. degree from University of New South Wales, Australian Defense Force Academy, Canberra, Australia, in 1996.

Following a research position at the same institution, he joined the University of Newcastle, Callaghan, Australia, in 1997, where he is currently an Associate Professor with the School of Electrical Engineering and Computer Science, the Assistant Dean for Research (Engineering), the head of Laboratory for Dynamics and Control of NanoSystems, and an Associate Director of the ARC Centre for Complex Dynamic Systems and Control, an Australian Government Centre of Excellence. He has published two books, several edited volumes, and over 150 refereed articles in the areas of robust control and estimation, smart structures, active noise and vibration control, mechatronic systems, and nanotechnology.

Dr. Moheimani is an Associate Editor of several international journals including the IEEE TRANSACTIONS ON CONTROL SYSTEMS TECHNOLOGY and has chaired a number of international workshops and conferences. 